\title{
Review on Phonon Transport within Polycrystalline Materials
}

\author{
Qing $\mathrm{Hao}^{1, *}$ and Jivtesh Garg ${ }^{2}$
}

\begin{abstract}
As one fundamental problem in materials science research, thermal transport across grain boundaries is critical to many energy-related applications. Due to the complexity of grain boundaries, the current understanding on how a grain boundary interacts with heat carriers is still in its infancy. This review summarizes the current progresses of this important topic, with its further extension to general interfaces. One focus is on major modeling and simulation techniques to predict the thermal resistance of a grain boundary. The corresponding thermal measurements of individual grain boundaries and grain-boundary thermal engineering are also discussed. A better understanding of the grain-boundary phonon transport is critical to many energy-related applications, where the concerned thermal transport within a polycrystalline material can be largely suppressed by grain boundaries.
\end{abstract}

Keywords: Grain boundaries; Interfacial thermal resistance; Phonon; Polycrstalline materials.

Received: 29 April 2021; Accepted: 07 June 2021.

Article type: Review article.

\section{Introduction}

Bulk and thin-film materials with nano- to micro-grains are widely used in various research fields. Applications include thermoelectric materials for power generation and refrigeration, ${ }^{[1-4]}$ thermal insulation, ${ }^{[5]}$ structural materials, ${ }^{[6]}$ thin-film solar cells, ${ }^{[7]}$ and electronic devices. ${ }^{[8,9]}$ In these materials, a high volumetric density of grain boundaries (GBs) can be found as the interface between crystalline regions as single grains. With a high volumetric density of GBs, phonon transport within these materials is strongly suppressed by the GB transmission or reflection of incident phonons, leading to an interfacial thermal resistance $R_{K}$ known as the Kapitza resistance. ${ }^{[10,11]}$ The reduction of the lattice thermal conductivity $\left(k_{L}\right)$ by GBs has been acknowledged for a long term and has been exploited to improve thermal insulation and thermoelectric materials. ${ }^{[1-3]}$ In other cases, efforts are also dedicated to reducing $R_{K}$ for GBs and general interfaces to facilitate heat spreading in electronic devices. ${ }^{[12]}$ As input parameters for general phonon transport analysis, bulk phonon mean free paths (MFPs) of important materials can be accurately determined by either first-principles calculations or measurements. ${ }^{[13,14]}$ However, $R_{K}$ predictions for interfaces such as GBs are still difficult because $R_{K}$ is largely affected by

\footnotetext{
${ }^{1}$ Department of Aerospace and Mechanical Engineering, University of Arizona, Tucson, AZ 85721, USA.

${ }^{2}$ Department of Aerospace and Mechanical Engineering, University of Oklahoma, Norman, OK 73019, USA.

*Email: qinghao@email.arizona.edu (Q. Hao)
}

the exact atomic structure of a real GB, which is a less ordered layer of a finite thickness. In this review, major advancements of GB studies are summarized for both fundamental phonon transport studies and thermal modeling of polycrystalline materials. Some future research directions are discussed on how to better engineer the GB phonon transport.

\section{Atomic structure of GBs - twist and tilt GBs}

In 1912, Rosenhain and Ewen first proposed the microscopic structure of GBs before the exact atomic structure was observed with an electron microscope. In their speculation, the interstitial space between misoriented grains is filled with uncrystallized atoms as "amorphous cement." ${ }^{[15]}$ In contrast, an ordered boundary model was proposed by Hargreaves and Hill, in which all atoms at a GB were hypothesized to be associated with the crystal on one side of the interface, i.e., no randomly added atoms. For now, it is understood that neither of these two models can accurately describe a GB. A more accurate description was given by Read and Shockley who recognized that the misorientations at a GB can be accommodated by dislocations. ${ }^{[16]}$ The density of these dislocations can be predicted by Frank's formula. ${ }^{[17]}$

For thermal transport, one important feature for the GB atomic structure is the misorientation between two adjacent grains. For symmetrical GBs consisting of regularly spaced edge dislocations, Klemens suggested that the scattering rate by a GB was inversely proportional to the square of the GB misfit angle. ${ }^{[18]}$ In general, GBs can be categorized according to extent of misorientation between two grains as low- and 
high-angle GBs. Low-angle GBs have a misorientation less than about 15 degrees. They consist of a set of dislocations with their properties being a function of the misorientation. High-angle GBs are characterized by misorientation greater than about 15 degrees. Properties of high-angle GBs are often assumed to be independent of the misorientation though some recent measurements do not support this when the strain field at the GB can largely impact the phonon transport. ${ }^{[1920]}$

GBs can be further classified as tilt or twist boundaries. A tilt boundary is where the rotation axis is parallel to the boundary plane (Fig. 1a). This boundary can be formed from a single, contiguous crystallite or grain which is gradually bent by an external force. The increase in energy related to the elastic bending of the lattice can be reduced by inserting dislocations. As the grain is bent further, increasing number of dislocations are introduced to accommodate the deformation, resulting in a low-angle boundary. The grain can now be considered to have split into two sub-grains of notably different orientations.

Another GB type is a twist boundary, where the misorientation occurs around an axis that is perpendicular to the boundary plane (Fig. 1b). These concepts of tilt and twist boundaries represent somewhat idealized cases. The majority of boundaries are of a mixed type, containing dislocations of different types and Burgers vectors, in order to create the best fit between the neighboring grains. Fig. 1c displays the (a)
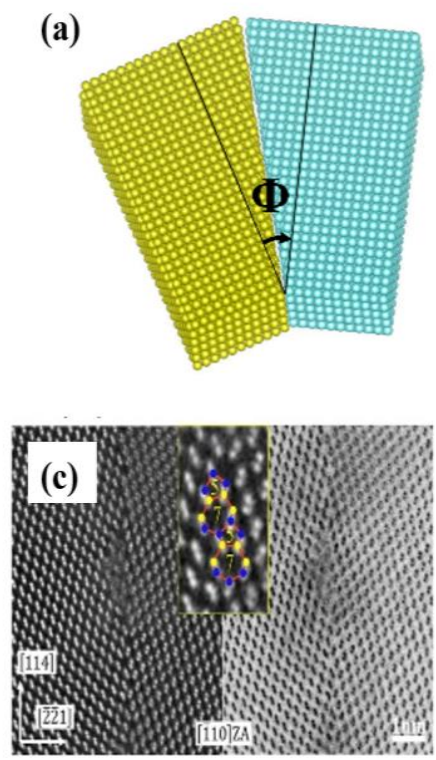

(b)
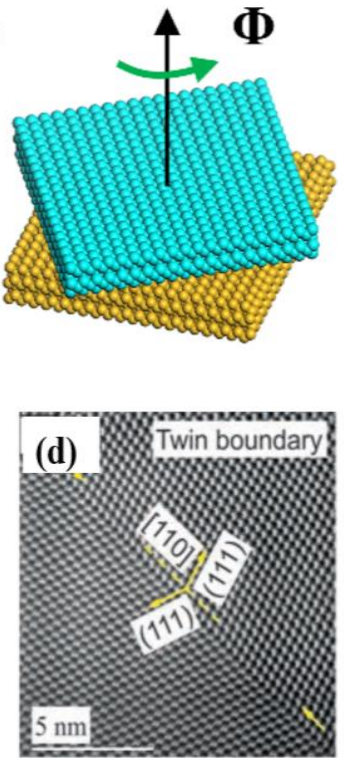

Fig. 1 Illustration of a typical (a) tilt GB and (b) twist GB. (c) High-angle annular dark-field TEM (left) and bright-field-TEM (right) images of a Si GB. The insets show a close-up of the respective $\mathrm{GB}$ atomic structures where two same color $\mathrm{Si}$ atoms (blue or yellow) share a bond on the same (110) plane, while two $\mathrm{Si}$ atoms with different colors share a bond on two consecutive (220) planes. (Reprinted with the permission from [21], Copyright 2020 American Institute of Physics). (d) TEM image of a twin boundary. (Reprinted with the Permission from [22], Copyright 2017 Elsevier B.V.) high-resolution transmission electron microscopy (TEM) images for a typical GB. As a special case, a twin boundary is shown in Fig. 1d, as a highly symmetrical interface.

3. $R_{K}$ of GBs and general interfaces - Analytical models, atomistic simulations, and machine learning predictions

In 1936, the existence of an interfacial thermal resistance $R_{K}$ was first found between liquid helium and a solid, in which an interfacial temperature jump $\Delta T$ occurred under an interface

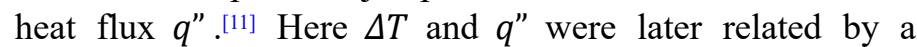
proportional constant $R_{K}$, i.e., $\Delta T=R_{K} q^{\prime \prime}$. The reciprocal of $R_{K}$ is the interfacial thermal conductance. Within the Landauer formalism, $R_{K}$ is determined by the phonon properties of both materials and the phonon transmissivity $\tau_{12}$ from Material 1 to Material 2. ${ }^{[11,23]}$ Assuming an isotropic $\tau_{12}$, simple analysis gives $R_{K}=4 / C_{1} v_{1} \tau_{12}$, in which $C_{1}$ and $v_{1}$ are the phonon specific heat and averaged phonon group velocity of Material 1, respectively. ${ }^{[23]}$ Approximating $C \sim 10^{6} \mathrm{~J} / \mathrm{m}^{3} \cdot \mathrm{K}$, $\tau_{12} \sim 0.1-1$, and $v \sim 10^{3} \mathrm{~m} / \mathrm{s}$, then the estimated $R_{K} \sim 1-$ $10 \times 10^{-9} \mathrm{~m}^{2} \cdot \mathrm{K} / \mathrm{W}$ is consistent with the data for nearly perfect interfaces. ${ }^{[24]}$ For general GBs with more interfacial defects, the transmissivity $\tau_{12}$, denoted as $\tau_{G B}$ for GBs, is reduced and $R_{K}$ becomes larger.

Although $C$ and $v$ can be easily determined from the phonon dispersion, $R_{K}$ based on the Landauer formalism is still difficult to be predicted due to the challenge in accurately computing $\tau_{12}$, particularly when the $\tau_{12}$ dependence on phonon angular frequency $\omega$ is considered. In practice, two major models are employed to compute $\tau$ across an ideal interface, namely the acoustic mismatch (AM) model and the diffuse mismatch (DM) model. These two models are briefly introduced below, with a focus on their restrictions for GBs. A more detailed review is given by Monachon et al. ${ }^{[25]}$ and Swartz et al. ${ }^{[11]}$

First, the AM model considers perfectly smooth interfaces so that specular and elastic phonon scattering (Fig. 2a) occurs on an interface. ${ }^{[26-28]}$ The predicted $\tau$ depends on the incident angle $\theta$, the sound velocity $c$ and mass density $\rho$ of both materials. For normal incidence, the transmissivity is $\tau_{12}=$ $4 Z_{1} Z_{2} /\left(Z_{1}+Z_{2}\right)^{2}$, in which $Z=\rho c$ is the acoustic impedance. The $\tau_{12}$ dependence on the phonon angular frequency $\omega$ is not considered here but is shown in modified AM models. ${ }^{[29,30]}$ In reality, the "smooth interface" condition for the AM model is only satisfied when the phonon wavelength is much longer than the interface roughness. This is the case only below $10 \mathrm{~K}$, when low-frequency phonons with long wavelengths become dominant for heat transfer. ${ }^{[11]}$ For a GB with the same material on both sides, the dilemma lies in that $100 \%$ phonon transmissivity $\tau_{G B}$ is expected due to no acoustic mismatch, which is clearly not reasonable.

For rough interfaces, Swartz and Pohl developed the DM model to compute the phonon transmissivity. ${ }^{[3]]}$ It assumes diffusive and elastic phonon scattering on an interface (Fig. $2 b)$. Phonons are assumed to lose their memory for which side they come from. In this case, $\tau_{12}$ is determined by the phonon 

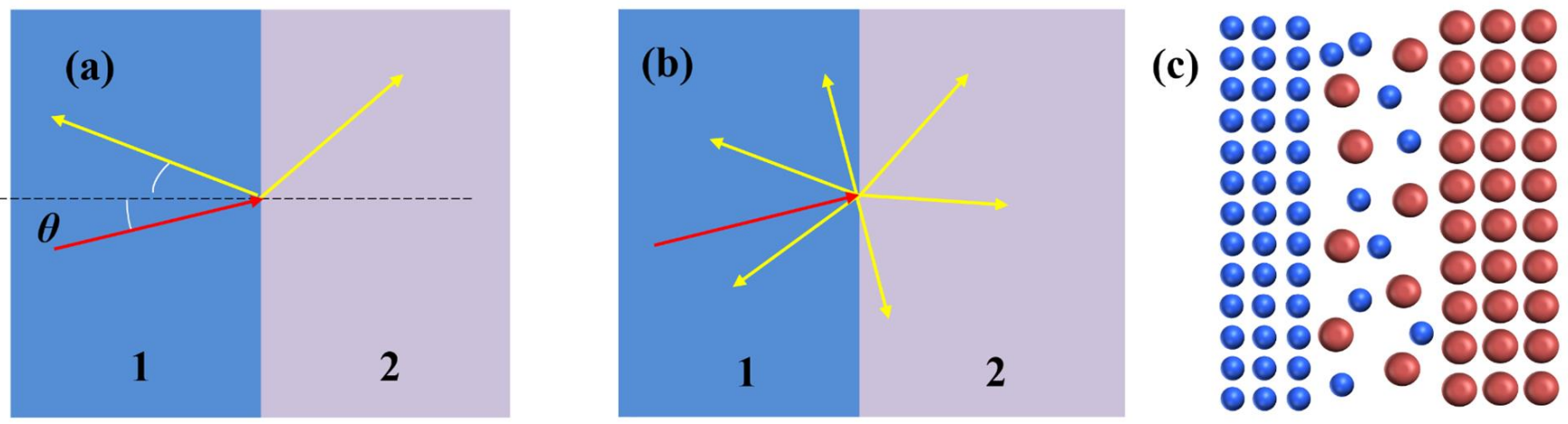

Fig. 2 (a) Specular scattering in the AM model. (b) Diffuse scattering assumed in the DM model. (c) Real interface simulated by MD simulation or AGF analysis.

group velocity and phonon density of states (DOS) within both materials. ${ }^{[11,32,33]}$ The frequency-dependent transmissivity from Material 1 to Material 2 is given as

$$
\tau_{12}(\omega)=\frac{\sum_{p} v_{g, p, 2}(\omega) D_{p, 2}(\omega)}{\sum_{p} v_{g, p, 1}(\omega) D_{p, 1}(\omega)+\sum_{p} v_{g, p, 2}(\omega) D_{p, 2}(\omega)},
$$

where $v_{g}$ is the phonon group velocity, $D$ is the phonon DOS, the subscript $p$ indicates the phonon branch, and $\omega$ is the phonon angular frequency. Assuming the Debye model with a constant $v_{g} \equiv v_{p}$ for branch $p$, Eq. (1) is simplified as

$$
\tau_{12}=\frac{\sum_{p} v_{p, 2}^{-2}}{\sum_{p} v_{p, 2}^{-2}+\sum_{p} v_{p, 1}^{-2}} \text {. }
$$

For a GB, a constant $\tau_{G B}=50 \%$ is predicted for all phonons due to structure symmetry. ${ }^{[34]}$ In contrast, energy dependence of $\tau_{G B}$ is recognized in numerous molecular dynamics (MD) simulations. ${ }^{[35-37]}$ Other calculations can also be found for the atomistic Green's function (AGF) method. ${ }^{[38-43]}$ In data analysis, a simple expression of $\tau_{G B}(p, \omega)$ has been proposed for a $\mathrm{GB}:{ }^{[44]}$

$$
\tau_{G B}(p, \omega)=\frac{1}{\frac{\gamma \omega}{\omega_{\max }}+1},
$$

where $\omega_{\max }$ is the maximum phonon frequency and the constant $\gamma$ is treated as one fitting parameter. The frequency dependence of $\tau_{G B}$ in Eq. (3) is simply missing in the DM model.

In physics, both the AM and DM models view an interface as a plane to join two materials and only use the bulk properties of both materials to compute $\tau_{12}$. However, a real interface is typically a layer region with disorder, roughness, dislocations, and often atom intermixing (Fig. 2c). ${ }^{[45]}$ The "real" interface properties were considered in modified versions of the DM and AM models. ${ }^{[46-48]}$ Despite such improvements, other important factors are still not included in the AM and DM models, such as the crystal misorientations and interfacial strains. ${ }^{[19,20,49]}$ For instance, a twin GB (Fig. 1d), with symmetric atomic structures on both sides of a GB, is known to possess a much lower $R_{K}$ than any types of GBs with different crystal orientations, ${ }^{[22,50,51]}$ which cannot be explained with AM or DM models. Such twin GBs are often associated with solid-state phase transformations.
Particular attention was also paid to how a GB interacted with phonons. In the early work by Klemens in 1994, ${ }^{[52]}$ the phonon velocity was assumed to be reduced within this less compacted interfacial layer, leading to a GB phonon reflectivity scaling with $\omega^{2}$ at low frequencies and saturating above a critical frequency. In some theories, the GB phonon scattering is dominated by GB dislocations whose scattering rates should be added to other scattering mechanisms inside a grain to compute the lattice thermal conductivity. ${ }^{[20,53-55]}$ Considering phonon diffraction by dislocations, the phonon scattering rates were derived analytically. ${ }^{[18,20,56,57]}$ The dislocation-phonon interaction was also studied based on a fully quantized dislocation field, called a "dislon". ${ }^{[58]}$ Some calculations also revealed the importance of the interfacial strain field. ${ }^{[20,57-59]}$

As more advanced techniques, MD simulations ${ }^{[35-37,60]}$ and AGF analysis ${ }^{[38-43]}$ can provide more insights into the interfacial phonon transport. The disordered interface layer can be incorporated into such simulations though an oversimplified interfacial atomic structure is still used in most studies. The AGF analysis assumes elastic interfacial phonon scattering and the phonon dynamic equation is solved under the harmonic approximation. Computationally, the AGF analysis is much more effective than MD simulations that track the phonon wave-packet reflection or transmission by an interface to yield $\tau_{G B}$ for different branches, incident angles, and frequencies. However, these approaches are very complicated and can only be performed for very limited GBs, which cannot largely benefit the data analysis of real polycrystalline materials with billions of different GBs. In addition, these theoretical predictions can hardly be validated experimentally due to the challenges in preparing, measuring, and characterizing an interface in a systematic and highly reproducible manner.

Despite the limitation for atomistic simulations, some general trends can still be identified for GBs. In the literature, the earliest MD simulation on a GB was carried out by Maiti et al. for polycrystalline $\mathrm{Si}^{[61]}$ The $R_{K}$ at a GB was extracted based on the simulated interfacial temperature drop and applied heat flow. At $575 \mathrm{~K}$, the averaged $\tau_{G B}$ was 0.65 and 
0.57 for the $\Sigma=5$ and the $\Sigma=13$ boundaries, respectively. Such $\tau_{G B}$ values were comparable to $\tau_{G B}=0.5$ for a GB, as predicted by Eq. (2). Li et al. ${ }^{[62]}$ computed the thermal conductance across silicon GBs with AGF analysis. They found that defective GBs significantly reduced the thermal transport. For a GB with dangling bonds, the thermal conductance was found to be diminished by more than 3-fold compared to case of pure silicon (Fig. 3a). For tilt GBs in $\mathrm{SrTiO}_{3}$, both acoustic and optical phonons were evaluated for their suppression by a GB. ${ }^{[37]}$ Although optical modes had a relatively lower transmissivity than acoustic phonons, they contributed to $50-60 \%$ of the thermal conductance across a GB. Beyond three-dimensional (3D) materials, the physics for phonon transport has also been revealed for GBs in twodimensional (2D) materials. Lu et al. ${ }^{[63]}$ studied the thermal conductance of GBs in graphene and found graphene GBs to exhibit a very high thermal conductance. The dependence of thermal conductance on the misorientation angle was found to be weak, with symmetric tilt zigzag GBs showing the best thermal conductance. Bagri et al..$^{[50]}$ studied the thermal transport across twin GBs in polycrystalline graphene from non-equilibrium MD simulations (Fig. 3b). They found the thermal conductance of twin GBs to be in the range of $1.5 \times$ $10^{10} \mathrm{~W} / \mathrm{m}^{2} \cdot \mathrm{K}$ to $4.5 \times 10^{10} \mathrm{~W} / \mathrm{m}^{2} \cdot \mathrm{K}$, significantly higher than any other thermoelectric interfaces reported in literature. Khalkhali et al..$^{[64]}$ investigated the thermal transport across GBs in polycrystalline silicene and found the average Kapitza conductance to be nearly $2.56 \times 10^{9} \mathrm{~W} / \mathrm{m}^{2} \cdot \mathrm{K}$ and $2.46 \times 10^{9}$ $\mathrm{W} / \mathrm{m}^{2} \cdot \mathrm{K}$ by utilizing the Tersoff and Stillinger Weber interatomic potentials, respectively. Strains were found to result in further changes in interfacial thermal conductance. Finally, it was found that by tuning the grain size of polycrystalline silicene, its thermal conductivity could be modulated up to one order of magnitude. ${ }^{[64]}$ Sandonas et al. ${ }^{[65]}$ studied the phonon transport properties of linear and curved graphene GBs under the influence of structural and dynamical disorder. To do this, density functional tight-binding (DFTB) method is combined with the AGF technique. The results show that curved GBs have a lower thermal conductance than linear GBs. The magnitude of thermal conductance depends on the length of the curvature and out-of-plane structural distortions at the boundary. In more recent studies, the phonondislocation scattering was also studied with MD simulations ${ }^{[66-}$ ${ }^{68]}$ to be compared with existing theoretical models. ${ }^{[18,20,56,57]}$ One MD simulation revealed that only phonons of a certain frequency range would be scattered by dislocations, ${ }^{[6]]}$ in contrast with a suppressed phonon MFP across the entire phonon frequency range.

In some analytical models, an interface is treated as a medium with its own properties and thickness, known as the virtual crystal assumption. ${ }^{[47]}$ Following this concept, more attention should be paid to the interfacial phonon modes. Recently, the interfacial modes of phonons are found by MD simulations, caused by the atomic level disorder close to the interface. ${ }^{[69-71]}$ The peaks in interfacial modes spectrum are broaden compared to those in bulk spectrum. ${ }^{[69]}$ In other words, the interfacial modes could take diffusive scatterings, which contribute to the interface resistance and nonlinear temperature profile close to interface.

In recent years, machine learning models have been proposed as an effective way to predict interfacial $R_{K}$, using available computational and experimental data for the training dataset. ${ }^{[72-75]}$ In this aspect, one obstacle is to find a robust and physically intuitive structure descriptor to describe different GBs or more general interfaces. In MD simulations, the GB excess volume, as a measure of non-optimum packing of interfacial atoms, was found to be strongly correlated with $R_{K} \cdot{ }^{[76]}$ Without other atomic information, however, this GB excess volume alone may not distinguish two GBs with possibly very different $R_{K}$ values. Keeping this in mind, the GB excess volume cannot be an accurate descriptor for machine learning models. Rosenbrock et al. ${ }^{[77,78]}$ used the

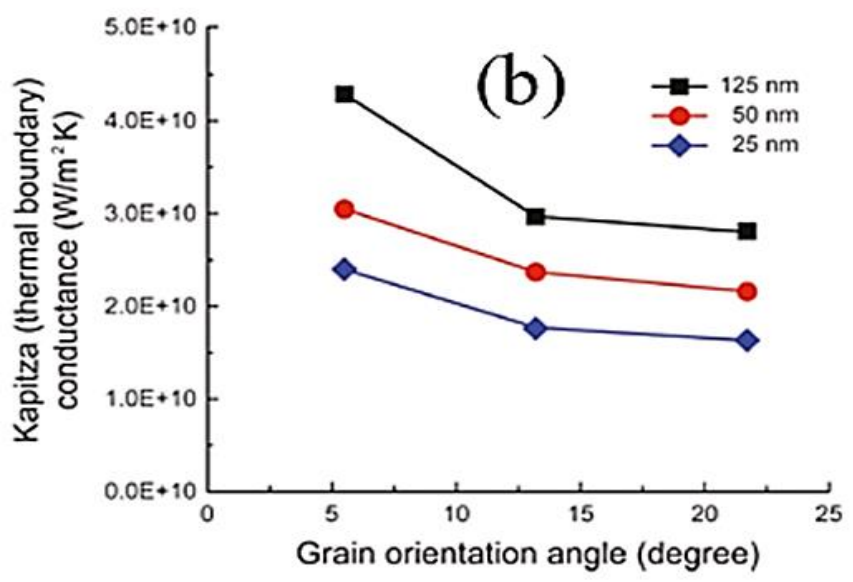

Fig. 3 (a) Interface thermal conductance across Si GBs as a function of the number of dangling bonds. (Reprinted with the permission from [62], Copyright 2019 Frontiers Media S.A.) (b) Dependence of Kapitza conductance in graphene on grain orientation angle. (Reprinted with the permission from [58], Copyright 2011 American Chemical Society) 
smooth overlap of atomic positions (SOAP) descriptor and identified a set of representative local atomic environments (LAEs) as building blocks to construct a GB. Following this, Fujii et al..$^{[74]}$ found small distortions to LAEs can be critical to the thermal conductivity. They demonstrated that the local distortion factor can correlate well with the predictions by MD simulations on $\mathrm{MgO}$ GBs. More studies should be carried out for better representative descriptors in the future.

\section{Analytical models for $k_{L}$ predictions of polycrystalline materials}

\subsection{A brief review of existing analytical models}

For general nanostructures, the lattice thermal conductivity $\left(k_{L}\right)$ is reduced due to decreased phonon MFPs. Here $k_{L}$ is computed by the kinetic relationship:

$$
k_{L}=\frac{1}{3} \sum_{p} \int_{0}^{\omega_{\max , p}} c_{p}(\omega) v_{g, p}(\omega) \Lambda_{e f f, p}(\omega) d \omega,
$$

where $\omega$ is the angular frequency of phonons, $c_{p}(\omega)$ is the differential volumetric phonon specific heat, and $v_{g, p}(\omega)$ is the phonon group velocity. The subscript $p$ indicates the phonon branch and usually only three acoustic branches are considered. The effective phonon MFP is modified from the bulk phonon MFP $\Lambda_{b u l k}$ by the characteristic length $\Lambda_{b d y}$ of the nanostructure. Here the Matthiessen's rule gives $\Lambda_{e f f, p}(\omega)=\left[1 / \Lambda_{b u l k, p}(\omega)+1 / \Lambda_{b d y}\right]^{-1}$. In early studies, Eq. (4) is used to compute the lattice thermal conductivity of polycrystalline materials and $\Lambda_{b d y}$ is simply the grain size $d$ when completely diffusive phonon scattering is assumed for GBs. ${ }^{[34,79-81]}$ This $\Lambda_{b d y}=d$ treatment is based on the Casmir limit proposed for the boundary scattering of phonons within small structures. ${ }^{[82]}$ In principle, the computed $k_{L}$ only represents the thermal conductivity of a single grain, instead of grains joined by GBs with an interfacial thermal resistance $R_{K}$. For grains with an intragranular lattice thermal conductivity $k_{G}$ and size $d$, an effective medium formulation $(\mathrm{EMF})$ suggests ${ }^{[83]}$

$$
k_{L}=\frac{k_{G}}{1+R_{K} k_{G} / 2 d},
$$

which can be further expanded as integration over phonon angular frequency and summation over different phonon branches. Assuming elastic and diffusive GB phonon scattering, the spectral $R_{K, p}(\omega)$ can be obtained by extending the expression derived under the gray-medium approximation $^{[84,85]}$

$$
R_{K, p}(\omega)=\frac{4}{\tau_{G B}(p, \omega) c_{p}(\omega) v_{g, p}(\omega)},
$$

where $\tau_{G B}(p, \omega)$ is the phonon transmissivity across a GB. Other $R_{K}$ expressions can also be found when phonon hopping is considered. ${ }^{[86]}$ The frequency-dependent form of Eq. (5) is simply[83,87,88]

$$
k_{L}=\sum_{p} \int_{0}^{\omega_{\max , p}} \frac{k_{G, p}(\omega)}{1+\frac{2 \Lambda_{e f f, p}(\omega)}{3 \tau_{G B}(p, \omega) d}} d \omega,
$$

in which $k_{G, p}(\omega)=c_{p}(\omega) v_{g, p}(\omega) \Lambda_{e f f, p}(\omega) / 3$ is the spectral lattice thermal conductivity of each grain. Equation (7) can further include the probability $P_{G B}$ for specular GB phonon transmission, which becomes critical at cryogenic temperatures. ${ }^{[87]}$ Existing EMF for polycrystalline structures are summarized in Table 1 but Eq. (7) is the only one validated with phonon MC simulations for the exact 3D structures (Fig. 4). More complicated cases such as a nanoparticle composite are modelled by Palla and Giordano. ${ }^{[89]}$ In addition, $k_{G}$ instead of the bulk lattice thermal conductivity $k_{B u l k}$ should be used in all the models to include the phonon size effects within small grains. ${ }^{[50,90]}$
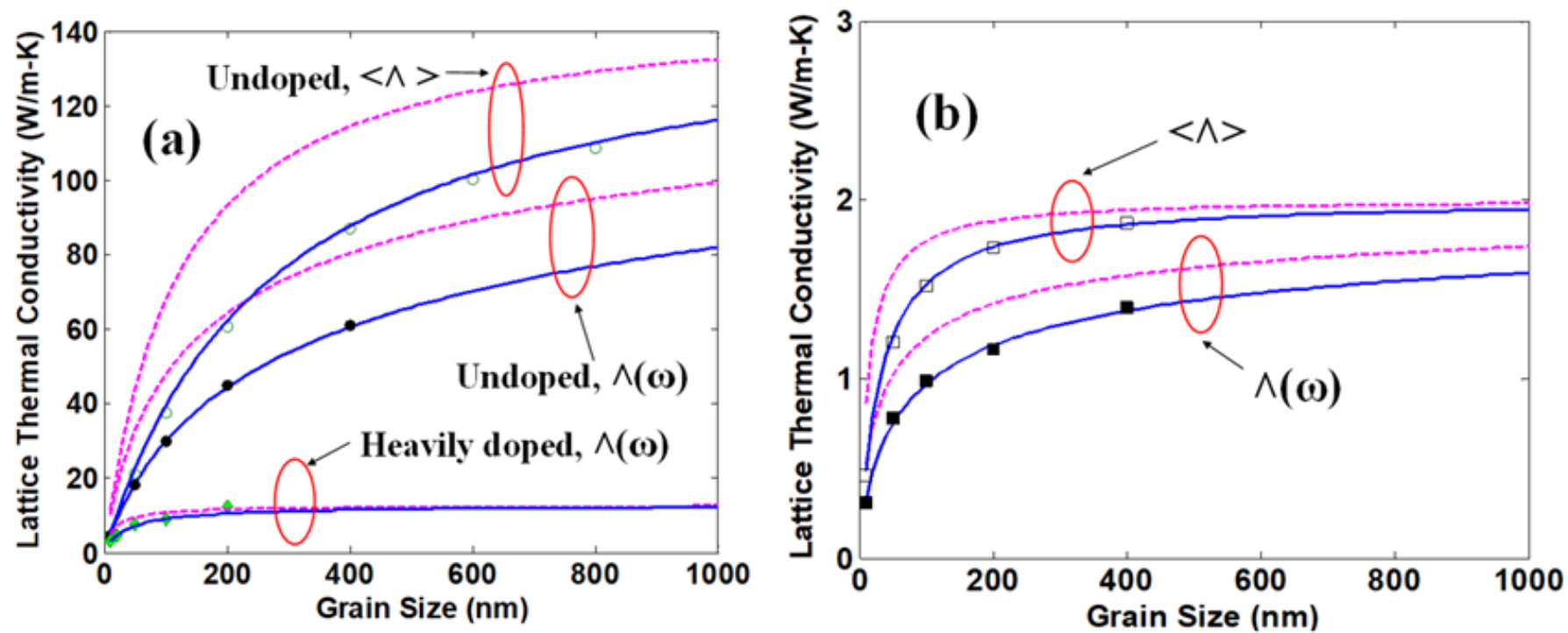

Fig. 4 Computed room-temperature $k_{L}$ values of selected polycrystals: (a) undoped and heavily doped silicon, (b) undoped PbTe. Three theoretical predictions are bundled together for either frequency-dependent or frequency-independent cases: kinetic relation in Eq. (1) (dashed lines), EMF (solid lines), phonon MC simulations (symbols). For frequency-independent analysis, $\langle\Lambda\rangle$ is marked on bundled curves, while $\Lambda(\omega)$ indicates frequency-dependent cases. (Reprinted with the permission from [83], Copyright 2012 American Institute of Physics) 
Table 1. Existing EMF for polycrystalline materials. In earlier models, a sharp GB with zero thickness is assumed. Badry and Ahmed ${ }^{[91]}$ further considered a GB as a thin layer with its thickness $l$ and conduction thermal resistance $R_{K}=\int_{0}^{l} \frac{d x}{k_{G B}(x)}$, with $k_{G B}$ as the local thermal conductivity within the GB layer.

\begin{tabular}{ll}
\hline Article & $k_{L}$ expression \\
\hline Yang $^{[5,92]}$ & $\frac{k_{G}}{1+R_{K} k_{G} / d}$ (derived for one-dimensional or \\
& $1 \mathrm{D}$ heat conduction) \\
Nan and Birringer ${ }^{[93]}$ & $\frac{k_{G}}{1+2 R_{K} k_{G} / d}$ \\
$\mathrm{Hao}^{[83]}$ & $\frac{k_{G}}{1+R_{K} k_{G} / 2 d}$ \\
Dong et al. ${ }^{[94]}$ & $\frac{k_{\text {Bulk }} /\left(1+\Lambda_{\text {bulk }} / d^{0.75}\right)}{1+R_{K}\left[k_{B u l k} /\left(1+\Lambda_{b u l k} / d^{0.75}\right)\right] / d}$ \\
Badry and Ahmed ${ }^{[91]}$ & $\frac{d+l}{d / k_{G}+R_{K}}$ \\
&
\end{tabular}

Other than EMF, simplified models can also be found for polycrystalline materials. Approximating the grained structure as a superlattice nanowire, ${ }^{[85]}$ the characteristic length $\Lambda_{b d y}$ of a polycrystalline material is modified from the grain size $d$ by ${ }^{[44]}$

$$
\frac{\Lambda_{b d y}}{d}=\left[\left(\frac{3}{4} \frac{\tau_{G B}}{1-\tau_{G B}}\right)^{-1}+1.12^{-1}\right]^{-1},
$$

which diverges from that given by ray tracing simulations by $\sim 30 \% .{ }^{[95]}$ However, both the ray tracing simulations and Eq. (8) still assume ballistic phonon transport within grains, i.e., no internal phonon scattering within a grain. When the derived $\Lambda_{b d y}$ is employed to modify $\Lambda_{b u l k}$ using the Matthiessen's rule, some errors are often anticipated because GB phonon scattering is a surface process and internal phonon scattering is a volumetric process. ${ }^{[23,96]}$ In this case, direct comparisons between predicted $k_{L}$ by the analytical model and phonon MC simulations for varied phonon MFP distributions and grain sizes are critical, which is addressed in the validation of Eq. (7). . $^{[83,87,88]}$

In practice, $\Lambda_{b d y}=\alpha d(0<\alpha<1)$ can be assumed to incorporate $R_{K}$ into Eq. (4) and $\alpha=0.63$ was fitted for the experimental data on polycrystalline $\mathrm{Bi}_{2} \mathrm{Te}_{3 .}{ }^{[97]}$ To better fit $k_{L}$ of nanocrystalline $\mathrm{Si}$ down to $\sim 30 \mathrm{~K}$ or below, $\Lambda_{b d y} \sim \alpha d / \omega$ in Eq. (4) is found to be accurate to include the energydependent GB phonon transmissivity and specularity, the latter of which becomes critical at cryogenic temperatures. ${ }^{[44}$ Different from single crystals with a $k_{L} \sim T^{3}$ trend at low temperatures, $k_{L} \sim T^{2}$ is often observed for polycrystalline materials ${ }^{[4,98,99]}$ and can be explained with $\Lambda_{b d y} \sim \alpha d / \omega$. Despite its simplicity, this frequency-dependent effective $\Lambda_{b d y}$ is in contrast with the widely used frequencyindependent $\Lambda_{b d y}$, which should be considered in the thermal design of a polycrystalline material. ${ }^{[81]}$

Other than above models, some crude models can also be found to estimate the grain-size dependent $k_{L}$. Here Takashiri et al. proposed a simple expression: ${ }^{[100]}$

$$
k_{L}= \begin{cases}k_{\text {Bulk }}-\frac{2}{3} k_{0} \sqrt{\frac{\langle\Lambda\rangle}{3 d}} & (\langle\Lambda\rangle \ll d) \\ \frac{2 k_{\text {Bulk }}}{3}\left[\frac{3 d}{\langle\Lambda\rangle}\left(\frac{k_{B u l k}}{2 k_{0}}\right)^{2}\right]^{1 / 4} & (\langle\Lambda\rangle \geq d)\end{cases}
$$

where $k_{B u l k}$ is the lattice thermal conductivity without the GB influence, $k_{0}$ is the lattice thermal conductivity without alloy scattering that corresponds to $\langle\Lambda\rangle$. Equation (9) employs the fact that GB tends to scatter long-MFP phonon and alloy scattering mainly affects short-MFP phonon.

\subsection{Grain size averaging}

In many polycrystalline materials, a wide grain size distribution is found and there exist questions for the effective grain size $d_{e f f}$ to be used in the modeling. The logarithmic normal distribution is often employed to describe the grainsize distribution function $f(\alpha)$, giving the percentage of grains with size $\alpha$ and $d \alpha$ accuracy: ${ }^{[101]}$

$$
f(\alpha)=\frac{1}{\alpha \delta \sqrt{2 \pi}} \exp \left\{-\left[\frac{\ln \left(\alpha / d_{\text {avg }}\right)}{\sqrt{2} \delta}\right]^{2}\right\},
$$

in which $d_{\text {avg }}$ is the mean grain diameter, $\delta$ is the standard deviation, and $\int_{0}^{\infty} f(\alpha) d \alpha=1$. Compared with the actual grain size distribution generated by the Voronoi diagram from scanning electron microscopy (SEM) images ${ }^{[55]}$ or detailed TEM studies, ${ }^{[102]} f(\alpha)$ in Eq. (10) may have a large divergence and more accurate $f(\alpha)$ is required for reliable thermal analysis. Fig. 5 shows the TEM image and actual grain size distribution of nanostructured bulk bismuth antimony telluride alloys, which requires more complicated $f(\alpha)$ to describe. ${ }^{[102]}$

With $f(\alpha)$ given, the averaged grain size can be determined by

$$
\frac{1}{d_{e f f}}=\frac{\int_{0}^{\infty} \alpha^{2} f(\alpha) d \alpha}{\int_{0}^{\infty} \alpha^{3} f(\alpha) d \alpha}
$$

Here the distribution function $f(\alpha)$ describes the percentage of grains at size $\alpha$, with $d \alpha$ accuracy. Equation (11) is derived by matching the volumetric interface area of a polycrystalline material with that for an ideal polycrystal with a uniform cubic grain size $d_{e f f}$. It has been shown that the volumetric interface area is the key parameter for the thermal conductivity reduction in nanostructured materials. ${ }^{[103-106]}$ Equation (11) is different from the averaged $d_{e f f}$ according to the averaged grain volume:[44]

$$
d_{e f f}^{3}=\int_{0}^{\infty} \alpha^{3} f(\alpha) d \alpha .
$$

In practice, the two ways to compute $d_{\text {eff }}$ do not give big divergence but Eq. (11) is more accurate in principle. Particular attention should also be paid to the thermally anisotropic polycrystals with more volumetric GB surface area perpendicular to certain driections to block the heat flow. In this situation, the direction-averaged $k_{L}$ can be estimated with $d_{e f f}$ given by Eq. (11). ${ }^{[103]}$

\subsection{Thermal anisotropy within each grain}

For some materials such as $\mathrm{Bi}_{2} \mathrm{Te}_{3}$, their layered lattice structures lead to strongly anisotropic phonon transport within both single crystals and grains. ${ }^{[107]}$ In estimation, the in-plane 

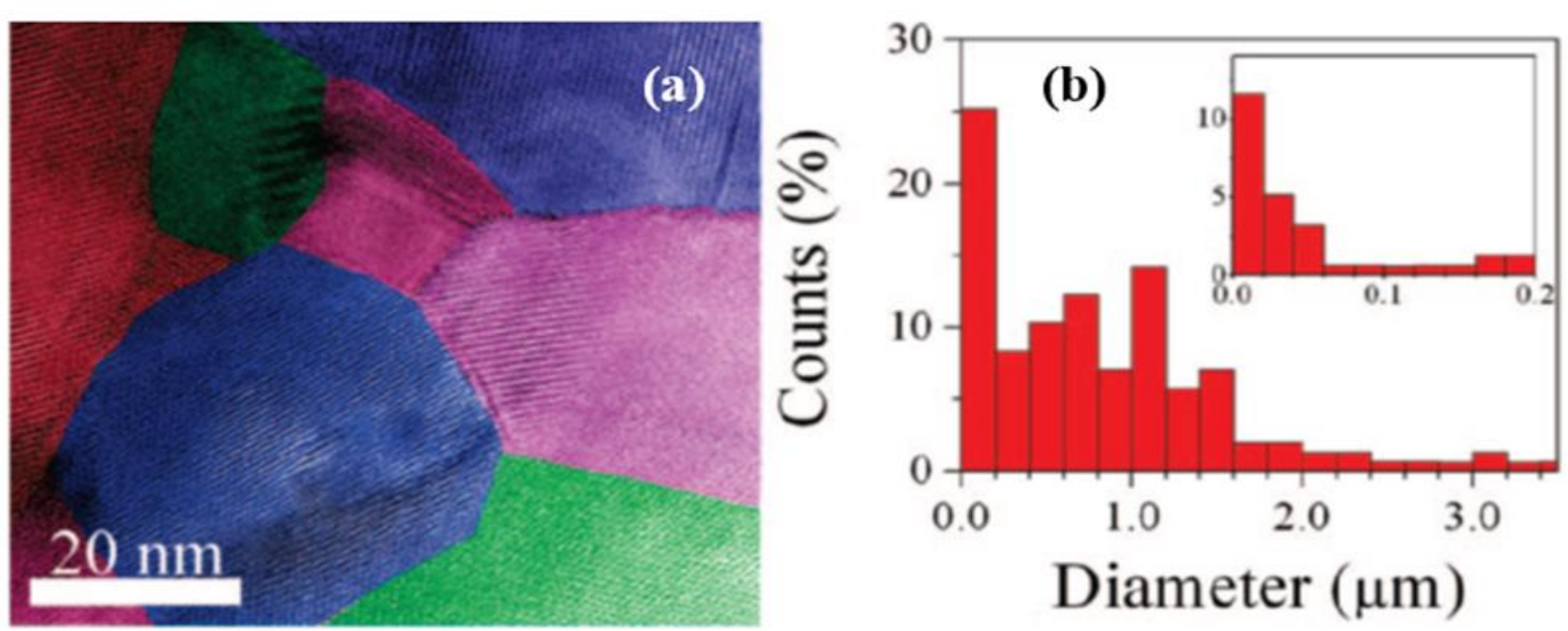

Fig. 5 (a) High-resolution TEM images of smaller grains, and (b) particle size distribution histogram for the nanograined bulk material with the inset zooming in on the distribution of small size particles less than $200 \mathrm{~nm}$. (Reprinted with the permission from [102], Copyright 2009 American Chemical Society)

thermal conductivity $k_{L, \|}$ of single-crystal $\mathrm{Bi}_{2} \mathrm{Te}_{3}$ is larger than its cross-plane lattice thermal conductivity $k_{L, \perp}$ by a factor of 2.1 at $300 \mathrm{~K} \cdot{ }^{[108]}$ It is thus important to average the lattice thermal conductivity for varied crystal orientations within different grains to find the effective $k_{L}$ along a given direction for a polycrystalline sample. When all grains are completely randomized for the crystal orientations and $R_{K}$ is negligible, the direction-averaged lattice thermal conductivity $\left\langle k_{L}\right\rangle$ is given by a "correlational approximation," as proposed by Mityushov and Adamesku:[109]

$$
\frac{\left\langle k_{L}\right\rangle}{k_{\text {char }}}=\frac{1}{3} r^{2 / 3}+\left[\frac{2}{3}-\frac{2}{9} \frac{(r-1)^{2}}{r+2}\right] r^{-1 / 3}
$$

with $r=\left\langle k_{L, \perp}\right\rangle /\left\langle k_{L, \|}\right\rangle, k_{\text {char }}=\left(\left\langle k_{L, \perp}\right\rangle\left\langle k_{L, \|}\right\rangle^{2}\right)^{1 / 3}$. Yang et al. ${ }^{[110]}$ compared different models for the directional average of $k_{L}$. It should be noted that Eq. (13) does not consider the critical $R_{K}$ and classical phonon size effects within each grain. One simple solution is to use Eq. (7) to compute the $k_{L}$ values corresponding to $k_{L, \|}$ and $k_{L, \perp}$. These two $k_{L}$ values can then be averaged with Eq. (13). More complicated EMF for nanograined materials with GB $R_{K}$ and anisotropic grains can be found elsewhere. ${ }^{[111]}$

In more complicated analysis, the orientation factor $F$ should be incorporated to consider the distribution of the grain orientations. $^{[112-116]}$ The orientation factor $F$ equals to 0 when grains are completely randomized for the crystal orientation; $F$ equals to 1 when the grains are highly oriented. For hotpressed samples, the layered atomic structure of each grain tends to be aligned along the direction that is perpendicular to the applied pressure. In this case, the direction average of $k_{L}$ must incorporate the impact of the orientation factor $F$.

\subsection{Grains with embedded nanostructures}

For thermoelectrics and other applications, hierarchical structures are often employed to suppress the lattice thermal conductivity across the whole phonon spectrum, ranging from alloy atoms as point defects to nano- and micro-structures as volumetric or interfacial defects. ${ }^{[3,117-120]}$ Here the scattering rate for point defects follow Rayleigh scattering, i.e., $\tau_{P D}^{-1} \sim \omega^{4}$. For dislocations as line defects, the dislocation core has $\tau_{D C}^{-1} \sim \omega^{3}$ and the strain field has $\tau_{D S}^{-1} \sim \omega \cdot{ }^{\left[{ }^{[1,121]}\right.}$ In modeling, an array of dislocations at an interfaces can be equally treated as a sum of individual dislocations within each grain. ${ }^{[55]}$ Attention should also be given to $\Lambda_{b d y} \sim \alpha d / \omega$ proposed by Wang et $a l .{ }^{[44]}$ after the impact of the frequency-dependent phonon transmissivity and specularity is incorporated into $\Lambda_{b d y}$. As a more accurate model, Eq. (7) should be used when an accurate $\tau_{G B}(p, \omega)$ is available. At cryogenic temperatures, specular GB phonon scattering also becomes important and the modified version of Eq. (7) should be used. ${ }^{[87]}$

In typical nanostructured bulk materials, nanoparticles may be embedded within nano- to micro-grains. Such nanoparticles can be dispersed nanoparticles or nanoprecipitates. Depending on the size and interface conditions of these nanoparticles, the scattering rate may interpolate between the long and short wavelength regimes, e.g., from $\tau_{N P}^{-1} \sim \omega^{0}$ for geometrical scattering limits to $\tau_{N P}^{-1} \sim \omega^{4}$ for Rayleigh scattering. ${ }^{[122-124]}$ Some optimization of the grain size distribution can thus be carried out to minimize the lattice thermal conductivity. ${ }^{[125]}$ When the particle-host interface is rough and diffusive phonon scattering is dominant, the treatment of nanoparticles can be simplified as that for a GB. As a simple approach, $\Lambda_{b u l k, p}(\omega)$ used in Eq. (7) should be replaced with an effective $\Lambda_{m, p}(\omega)$ for a bulk material with embedded nanoparticles but not GBs, given as ${ }^{[88,106,126]}$

$$
\Lambda_{m, p}(\omega)=\frac{k_{\text {particle }, p}(\omega)(1+2 \alpha)+2 k_{h, p}(\omega)+2 \varphi\left[k_{\text {particle }, p}(\omega)(1-\alpha)-k_{h, p}(\omega)\right]}{k_{\text {particle }, p}(\omega)(1+2 \alpha)+2 k_{h, p}(\omega)-\varphi\left[k_{\text {particle }, p}(\omega)(1-\alpha)-k_{h, p}(\omega)\right]} \Lambda_{h, p}(\omega),
$$


where $h$ indicates the host material, $\varphi$ is the volumetric fraction of embedded nanoparticles. When the embedded nanoparticles are replaced with nanopores, the lattice thermal conductivity of a particle, $k_{\text {particle }, p}(\omega)$, is simply zero in Eq.(14). Denote $R_{K, p}(\omega)$ as the spectral interfacial thermal resistance between a particle and the host and $d_{0}$ as the particle size. The dimensionless parameter $\alpha$ is

$$
\alpha=\frac{R_{K, p}(\omega) k_{h, p}(\omega)}{d_{0} / 2} .
$$

To incorporate classical phonon size effects with embedded nanostructures in a host material, $k_{\text {particle }, p}(\omega)$ and $k_{h, p}(\omega)$ should be both modified with the characteristic length that is $d_{0}$ for $k_{\text {particle }, p}(\omega)$. For $k_{h, p}(\omega)$, this characteristic length should be the radiative mean beam length (MBL): ${ }^{[127,128]}$

$$
M B L=\frac{4 V_{\text {Host }}}{S_{\text {Particle }}},
$$

where the $V_{\text {Host }}$ is volume for the host material and $S_{\text {Particle }}$ is the surface area of embedded nanoparticles. This MBL can be evaluated for one period of a nanocomposites and $V_{\text {Host }}$ is the volume after subtracting the nanoparticle volume. For nanoporous materials, other characteristic lengths proposed for phonons scattered by nanostructures like nanopores within a host can be found elsewhere. ${ }^{[155,106,129-132]}$ This analytical model is validated by simulating a structure with eight $\mathrm{Si}$ nanoparticles uniformly distributed within equal-sized cubic Ge grains (Fig. 6). ${ }^{[88]}$ Good agreement is found between phonon MC simulations (symbols) and theoretical modeling (lines).

5. Thermal measurements and thermal engineering of GBs Although thermal transport within polycrystalline thin films and bulk materials have been widely studied over the years, the current understanding of phonon transport across a single
GB is still very limited. Less information can be provided by the measured thermal conductivities for a sample with billions of different GBs with varied interfacial defects and crystal orientations. On the other hand, challenges lie in measuring a single GB within a 3D bulk material. Experimentally, $R_{K}$ of nonmetal-nonmetal interfaces are either directly measured for the interface between a thin film and its grown substrate, ${ }^{[24,133-}$ ${ }^{135]}$ or extracted from analyzing the measured cross-plane $k$ of a multilayered thin film. ${ }^{[136]}$ For fundamental studies, the impact of interfacial defects and other factors can be examined and the measured $R_{K}$ can be directly compared with model predictions or between different interfaces. ${ }^{[135]}$ These thin films, formed by epitaxy/hetero-epitaxy growth or deposition, may have quite different interface properties from those in a nanostructured bulk material that is often synthesized by hot pressing nanoparticles into the bulk form..$^{[1,79,80,137]}$ Therefore, the developed $R_{K}$ modeling may not be applicable to general nanostructured materials synthesized by different techniques. To better understand GBs formed by hot pressing nanopowder into a bulk material, wafer-wafer bonding has been used to represent a twist GB with relative rotation between identical crystals on the two sides of a GB. ${ }^{[138,139]}$ Thermal measurements for such a 1D interface was used to gain insights into the phonon transport across a single GB. One major problem for such thermally bonded interface is that the interface quality may not be maintained across a large wafer, where separation or fracture can be found at the interface. To address this problem, $\mathrm{Xu}$ et al. used super-flexible thin films hot pressed onto a wafer to represent a twist GB. ${ }^{[19]}$ The obtained high-quality interfaces can provide more reliable $R_{K}$ values for thermal studies. Figs. $7 \mathrm{a}$ and $7 \mathrm{~b}$ display the crosssectional and plane-view TEM images of a GB with a $3.4^{\circ}$ twist angle, respectively. In Fig. 7a, an "amorphous" 3-nm-
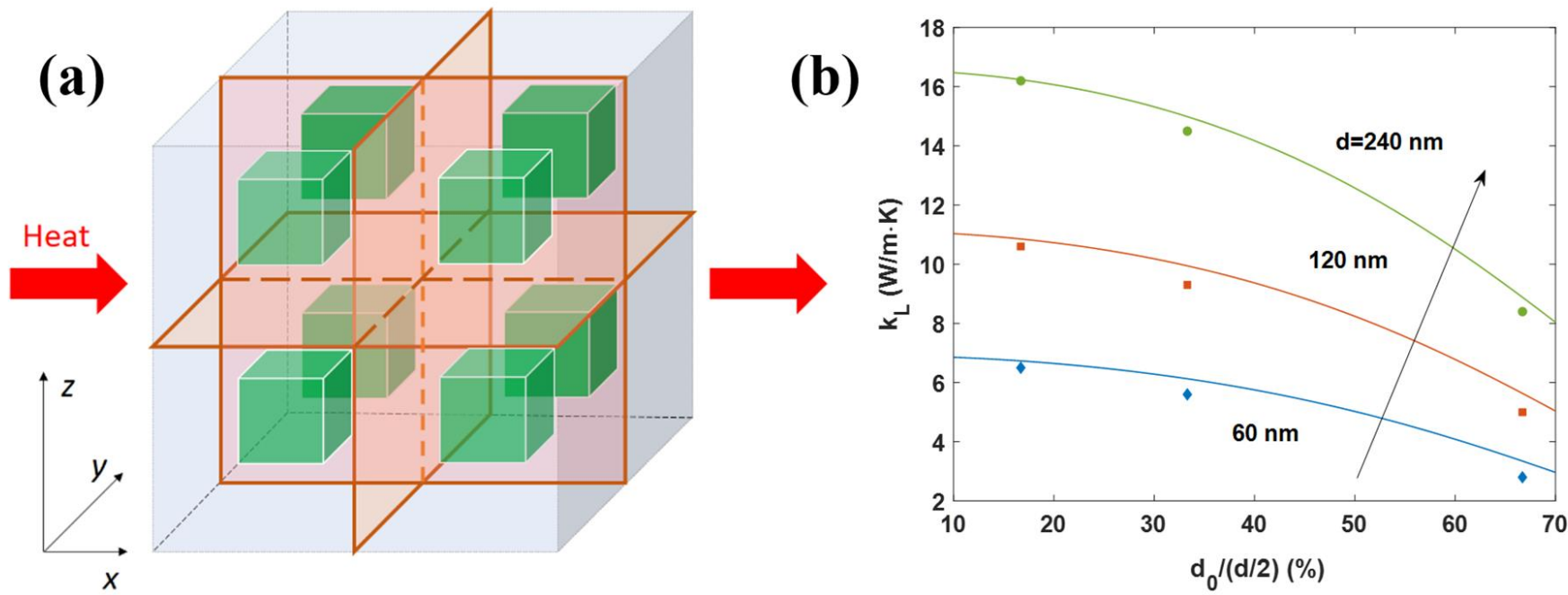

Fig. 6 (a) Computational domain of the simulated periodic structure, showing eight adjacent $1 / 8$ grains. The grain boundaries are three perpendicular planes. Each grain has eight embedded nanoparticles. (b) Comparison between the EMF (lines) and phonon MC simulations (symbols) for nanograined bulk Ge within eight equal-sized Si nanoparticles within each grain. Representative grain size $d$ and particle size $d_{0}$ are considered here for room-temperature calculations. Here the period for the nanoparticle array is simply $d / 2$. (Reprinted with the permission from [88], Copyright 2018 American Institute of Physics) 

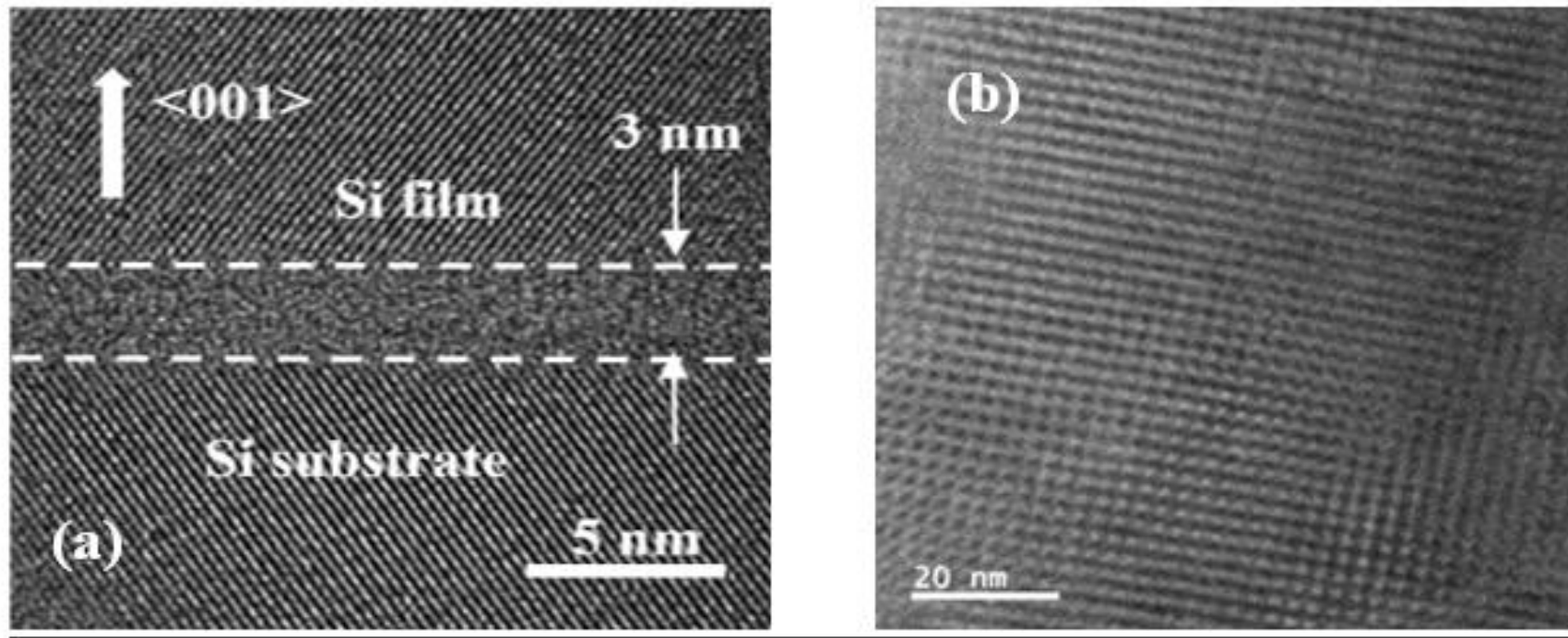

Fig. 7 (a) Cross-sectional TEM images of a 70-nm-thick Si film bonded onto a Si substrate by hot press, with a twist angle of $3.4^{\circ}$ after $1173 \mathrm{~K}$ anneal. The GB region is enclosed by dashed lines, with the thickness indicated. (b) Plane-view TEM image for the GB. (Reprinted with the permission from [19], Copyright 2018 Elsevier B.V.)

thick interface layer is found. Instead of an amorphous layer, more careful examination in Fig. $7 \mathrm{~b}$ shows a dislocation network across the GB. More discussions on the GB structures can be found elsewhere. ${ }^{[140,141]}$

Figure 8 summarizes the twist-angle-dependent thermal resistance of measured interfaces. Particular attention should be paid to the strong correlation between $R_{K}$ and the strain part $\gamma_{\text {strain }}$ of the total GB energy $\gamma_{G B}=\gamma_{\text {strain }}+\gamma_{\text {core }}$. Here the core energy $\gamma_{\text {core }}$ is associated with broken bonds across an interface. These two parts for $\gamma_{\mathrm{GB}}$ can be modeled by the extended Read-Shockley model proposed by Wolf, ${ }^{[142]}$ which was based on the well-known Read-Shockley model. ${ }^{[16]}$ Different from the observation here, MD simulations ${ }^{[36,143,144]}$ often suggest that $R_{\mathrm{K}}$ is correlated with the $\gamma_{\mathrm{GB}}$ and a larger $\gamma_{\mathrm{GB}}$ leads to a higher $R_{\mathrm{K}}$. The experimental results here instead suggest the importance of interfacial strain fields in suppressing the phonon transport, which is alignment with some calculations. ${ }^{[145]}$ The importance of $\gamma_{\text {strain }}$ for $R_{\mathrm{K}}$ is recently recognized for interfaces between $2 \mathrm{D}$ materials. ${ }^{[146,147]}$ For heterojunctions formed under the hot press, the interdiffusion between two dissimilar materials can further form an alloy interface that can largely increase the $R_{\mathrm{K}}{ }^{\left[{ }^{[148]}\right.}$

Despite decades of thermal studies on polycrystalline samples, few experimental studies can be found to reveal the $R_{\mathrm{K}}$ of a single GB. In an early study, in-situ TEM was used to measure $R_{\mathrm{K}}$ between two micron $\alpha-\mathrm{Al}_{2} \mathrm{O}_{3}$ fillers inside an epoxy resin, using a thermocouple tip for temperature measurements and an electron beam as a movable heating source ${ }^{[150]}$ For graphene grown by chemical vapor deposition (CVD), the GB $R_{\mathrm{K}}$ was determined from the difference between the thermal resistances of supported graphene with and without a GB. ${ }^{[151]}$ However, this simple subtraction to obtain $R_{\mathrm{K}}$ is still based on the Fourier's law and may not be valid when detailed phonon transport and the exact

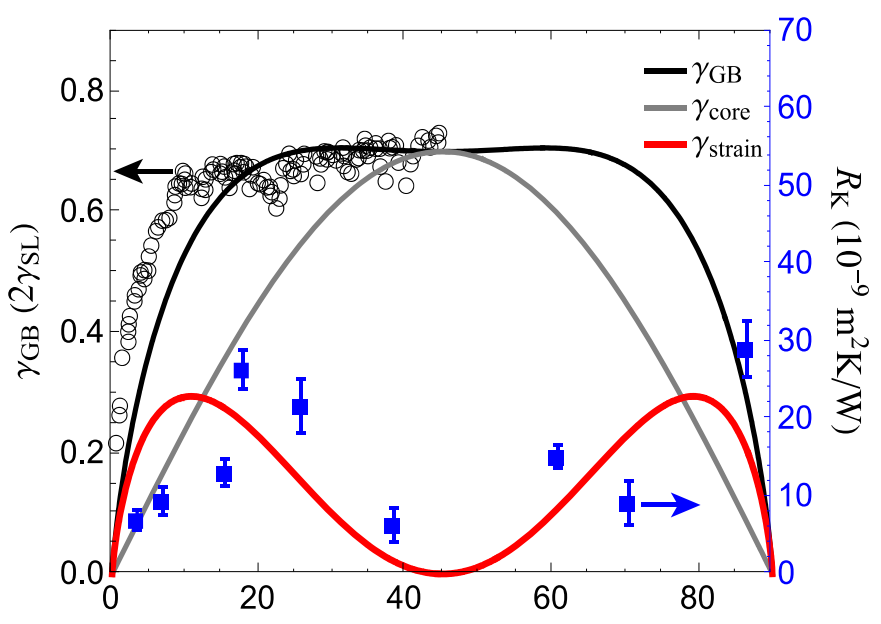

Twist Angle, $\theta\left(^{\circ}\right)$

Fig. 8 Experimental data of the GB energy $\left(\gamma_{\mathrm{GB}}\right)$ and thermal boundary resistance $\left(R_{\mathrm{K}}\right)$ of Si twist GBs. Open circles (left axis) are data from Otsuki ${ }^{[149]}$. Blue squares (right axis) are data from this study for samples after annealing. The lines are associated with the left axis and show the extended Read-Shockley model for GB energies, where the core energy $\left(\gamma_{\text {core }}\right)$ and the strain energy $\left(\gamma_{\text {strain }}\right)$ sum to give $\gamma_{\mathrm{GB}}$. (Reprinted with the permission from [19], Copyright 2018 Elsevier B.V.)

temperature distribution near a GB is considered. ${ }^{[23]}$ Using spatially resolved time-domain thermoreflectance (TDTR) measurements in combination with electron backscatter diffraction (EBSD), Sood et al. finds the local thermal conductivity can be reduced from $\sim 1000 \mathrm{~W} / \mathrm{m} \cdot \mathrm{K}$ at the center of large grains to $\sim 400 \mathrm{~W} / \mathrm{m} \cdot \mathrm{K}$ in the immediate vicinity of columnar GBs within boron-doped polycrystalline diamond (Fig. 9). ${ }^{[152]}$ Due to the spatial resolution, such measurements are still restricted to large grain size that is $\sim 25 \mu \mathrm{m}$ for the studied samples. The data analysis for a film with $\sim 530 \mu \mathrm{m}$ 


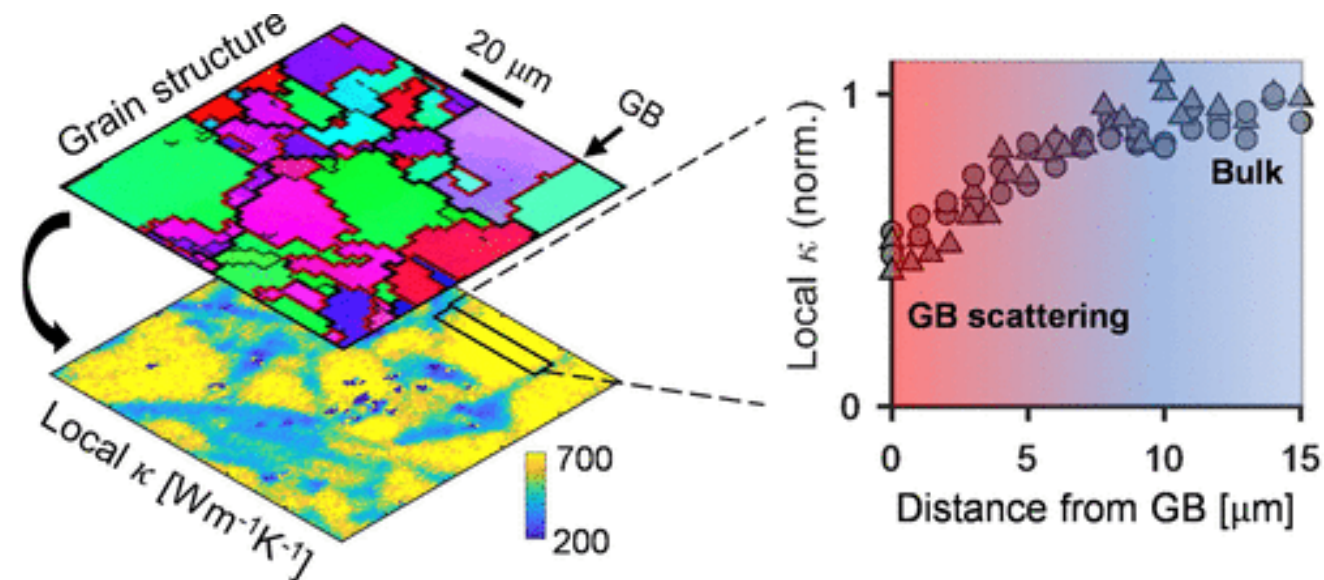

Fig. 9 Spatially resolved thermal conductivity measurements near individual GBs. Reprinted from Ref. [152]. Copyright 2018 American Chemical Society.

thickness and columnar grains can also be simplified as 1D thermal transport along the in-plane direction. When 3D micro-grains are measured, the film should be reduced to a single layer of grains and suspended to avoid any heat leakage into the substrate, which will add more challenges to the measurements.

Combined with thermal studies on bulk materials, there exists a continuous effort in tailoring the GB thermal transport to benefit certain applications. ${ }^{[153]}$ In the materials design, the nonequilibrium Green's function and machine learning algorithm can be combined to optimize the mass distribution of the interlayer, giving the maximum and minimum $R_{\mathrm{K}}$ values. ${ }^{[154]}$ To manipulate the $R_{\mathrm{K}}$ of general interfaces, numerous studies suggest the impact of interfacial roughness, ${ }^{[38,155-157]}$ nanostructure modification, ${ }^{[158-160]}$ isotopephonon scattering, ${ }^{[161]}$ and transition layers at an interface. ${ }^{[162,}$ ${ }^{163]}$ For thermoelectrics, a high electrical conductivity but a low thermal conductivity is preferred, leading to a high thermoelectric figure of merit (ZT). Here ZT is defined as $Z T=S^{2} \sigma T / k$, where $S, \sigma, k$, and $T$ represent Seebeck coefficient, electrical conductivity, thermal conductivity, and absolute temperature, respectively. ${ }^{[164]}$ Adding an interfacial layer is thus attempted to improve the thermoelectric performance of a bulk material if this interface does not negatively affect the electron transport. In this aspect, Ji et al. proposed GB engineering by hydrothermal coating of micro $\mathrm{Bi}_{2} \mathrm{Te}_{3}$ power with alkali-metal(s)-containing materials before hot press, leading to an interface layer to block the thermal transport. ${ }^{[165]} \mathrm{A} \sim 30 \% \mathrm{ZT}$ enhancement was observed for Natreated samples. Even without special coating, TEM studies ${ }^{[102}$ for high-ZT BiSbTe nanostructured bulk alloys ${ }^{[3]}$ also showed a 4-nm-thick bismuth-rich interface layer at GBs (Figs. 10a and $b$ ). When dopants are added beyond the solid solubility of the grains, they may also be accumulated at the GB to form an interface layer. ${ }^{[166]}$ In the study by Kim et al., ${ }^{[118]}$ dense dislocation arrays were formed at low-energy GBs during liquid-phase compaction in $\mathrm{Bi}_{0.5} \mathrm{Sb}_{1.5} \mathrm{Te}_{3}$ (Figs. 10c and d), which was suggested to effectively scatter midfrequency phonons and thus lower the $k_{L} \cdot{ }^{[118]}$ However, the ZT 1.86 reported by Kim et al. ${ }^{[118]}$ was not reproducible in the studies by Jo et al. ${ }^{[167]}$ and Deng et al. ${ }^{[168]}$ The contrast was attributed to the ZT overestimate with thermal and electrical properties measured along different directions for an anisotropic sample ${ }^{[168]}$ Along the direction perpendicular to the hot press direction, the minimum $k_{L}=0.65 \mathrm{~W} / \mathrm{m} \cdot \mathrm{K}$ was measured by Deng et al. ${ }^{[168]}$ and was almost twice as large as that reported by Kim et al. ${ }^{[18]}$ For PbTe, dislocations were found to be effective for $\mathrm{ZT}$ enhancement, where a $\mathrm{ZT}=2.2$ was achieved. ${ }^{[169]}$ More research is thus required to better understand these materials. In other studies, wrapping micrograins with graphene led to a $\mathrm{ZT}=1.5$ in n-type $\mathrm{Yb}_{y} \mathrm{Co}_{4} \mathrm{Sb}_{12}$, where the GB thermal resistance can be increased by a factor of 3 to 5, with a minor impact on the electron transport. ${ }^{[170]}$ Along this line, InSb nano-phase with a typical size of 5-15 $\mathrm{nm}$ was dispersed at GBs with grain sizes of 100-200 nm to achieve a ZT of 1.5 at $800 \mathrm{~K}$ for nanostructured $\mathrm{In}_{\mathrm{x}} \mathrm{Ce}_{\mathrm{y}} \mathrm{Co}_{4} \mathrm{Sb}_{12+z}{ }^{[171]}$ Similarly, $\mathrm{ZT}$ of 1.4 at $875 \mathrm{~K}$ in $\mathrm{CoSb}_{3-}$ based nanocomposites was also obtained using $\mathrm{Yb}_{2} \mathrm{O}_{3}$ nanoprecipitates and CNTs to modify the GBs. ${ }^{[172]}$

\section{Summary and perspective}

With its complexity, interfacial thermal transport is still not well understood even after decades of research. Due to the challenge in measuring individual 3D GBs within a material, the computed $R_{K}$ can hardly be compared with real measurements and available $R_{K}$ data are often for a planar interface within grown thin films. Such planar interfaces have less defects and can differ greatly from the non-planar and more complicated GBs within hot-pressed bulk materials or CVD polycrystalline thin films. In this aspect, the defects associated with the synthesis conditions are not addressed. Some recent MD simulations attempted to mimic the hot-press process to obtain more accurate interfacial atomic structures for the following phonon simulations. ${ }^{[41]}$ Unrestricted to thermal transport, MD simulations on the formation of GBs are widely available for the patterns of interfacial nanostructures. ${ }^{[173,174]}$ These atomic structures should be correlated with the actual interfacial atomic structure, as 

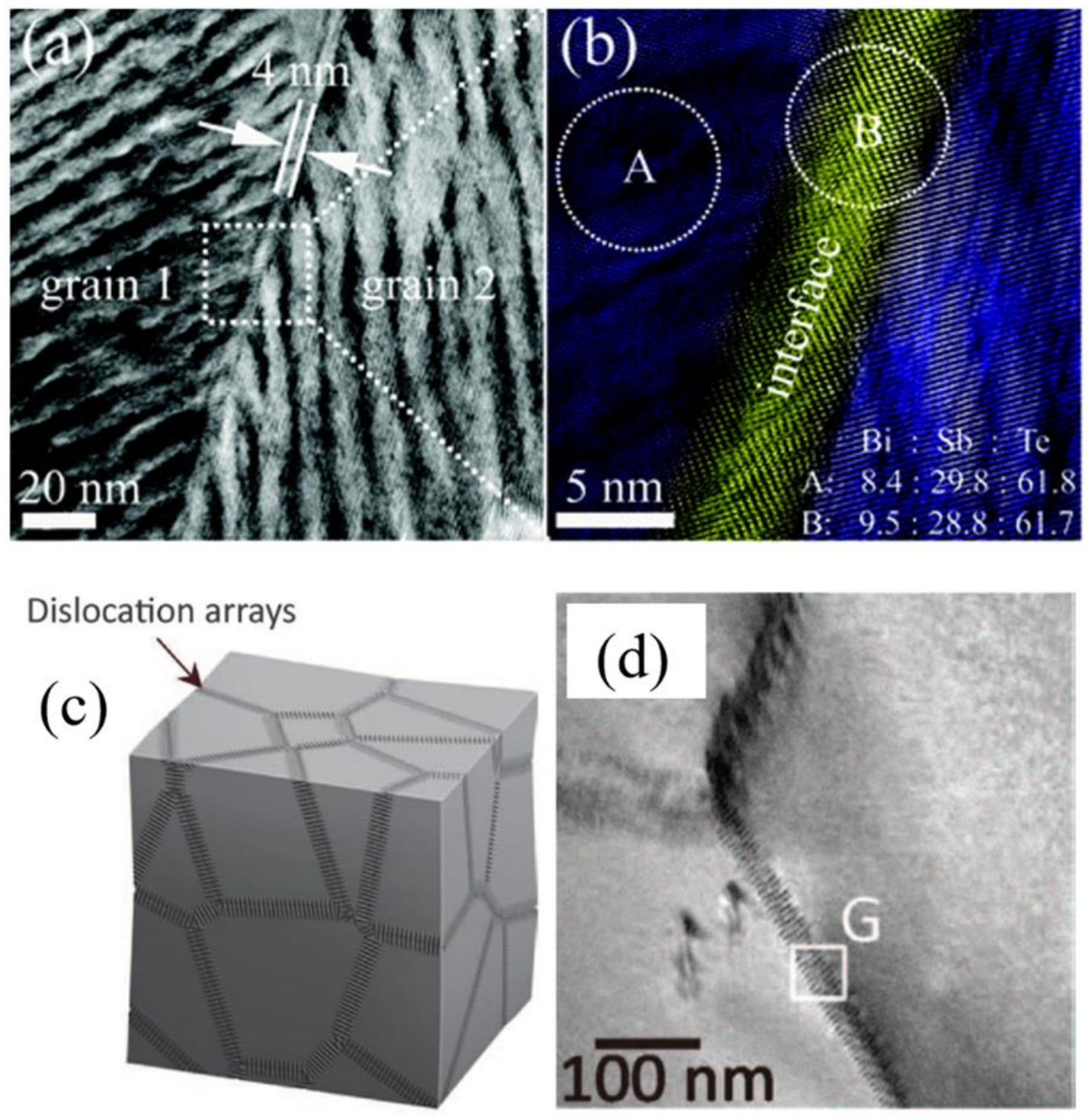

Fig. 10 (a) TEM and (b) high-resolution TEM image of a bismuth-rich interface region between two grains in the nanograined bulk material. The dotted circles indicate the regions from where energy-dispersive X-ray spectroscopy (EDS) spectra were measured (with the compositions for each region given in atomic percentage). Reprinted from Ref. [102]. Copyright 2009 American Chemical Society. (c) Dislocation arrays formed during the liquid-phase compaction process. The Te liquid (red) between the $\mathrm{Bi}_{0.5} \mathrm{Sb}_{1.5} \mathrm{Te}_{3}$ grains flows out during the compacting process and facilitates the formation of dislocation arrays embedded in low-energy GBs. (d) TEM image of a Te-MS material. Figures (c) and (d) are reprinted from Ref. [118]. Copyright 2015 The American Association for the Advancement of Science.

revealed with electron microscopy. For relatively simple materials systems, the phonon scattering by dislocations have recently been studied with MD simulations to compare with theoretical models. ${ }^{[66]}$ More studies should be further carried out in this direction, with an emphasis on the synthesisstructure-property relationships.

For engineering applications, there exist enormous research opportunities in controlling the interfacial nano- and atomic structure and thus adjusting the $R_{K}$. Beyond GBs, the heat dissipation within $\mathrm{GaN}$-based power electronics is largely hindered by the large interfacial thermal resistance between the GaN film and the substrate. ${ }^{[175]}$ Cooling techniques applied to the substrate side are thus less effective for heat removal, e.g., using an ultra-high-thermal-conductivity diamond substrate or adding cooling channeling within a substrate. ${ }^{[175-}$ ${ }^{178]}$ Along this line, some interfacial improvement on the phonon transport can be achieved by optimizing the interfacial nanostructure. ${ }^{[157,160,179-183]}$ On the other side, a deposited diamond film as a heat spreader on top of the device is also less useful when nanoscale columnar grains are found. ${ }^{[152]}$ New growth conditions to maximize the crystallization must be addressed to reach the full potential of diamond for thermal transport. GB migration during the synthesis ${ }^{[184]}$ should be better understood in this case.

\section{Acknowledgements}


Q.H. acknowledges the support from the National Science Foundation (grant number CBET-1651840) for phonon studies on GBs. J. G. thanks National Science Foundation (grant number 1847129).

\section{Conflict of Interest}

There is no conflict of interest.

\section{Supporting Information}

Not applicable.

\section{References}

[1] Y. Ma, Q. Hao, B. Poudel, Y. Lan, B. Yu, D. Wang, G. Chen and Z. Ren, Nano Lett., 2008, 8, 2580-2584.

[2] J. Yang, Q. Hao, H. Wang, Y. C. Lan, Q. Y. He, A. Minnich, D. Z. Wang, J. A. Harriman, V. M.Varki, M. S. Dresselhaus, G. Chen and Z. F. Ren, Phys. Rev. B, 2009, 80, 115329.

[3] B. Poudel, Q. Hao, Y. Ma, Y. Lan, A. Minnich, B. Yu, X. Yan, D. Wang, A. Muto and D. Vashaee, Science, 2008, 320, 634-638. [4] Q. Hao, D. Xu, N. Lu and H. Zhao, Phys. Rev. B, 2016, 93, 205206.

[5] H.S. Yang, G.R. Bai, L. Thompson and J. Eastman, Acta Mater., 2002, 50, 2309-2317.

[6] C. C. Koch, J. Mater. Sci.., 2007, 42, 1403-1414.

[7] J. Rath, Sol. Energy Mater. Sol. Cells, 2003, 76, 431-487.

[8] R. Sporea, T. Burridge and S. Silva, Sci. Rep., 2015, 5, 14058.

[9] I. R. Post, P. Ashburn and G. R. Wolstenholme, IEEE T. Electron Dev., 1992, 39, 1717-1731.

[10] P. L. Kapitza, J. Phys. (Moscow), 1941, 4, 181.

[11] E. T. Swartz and R. O. Pohl, Rev. Mod. Phys., 1989, 61, 605668.

[12] D. G. Cahill, P. V. Braun, G. Chen, D. R. Clarke, S. Fan, K. E. Goodson, P. Keblinski, W. P. King, G. D. Mahan, A. Majumdar, H. J. Maris, S. R. Phillpot, E. Pop and L. Shi, Appl. Phys. Rev., 2014, 1, 011305.

[13] T. Luo and G. Chen, Phys. Chem. Chem. Phys., 2013, 15, 3389-3412.

[14] T. Feng and X. Ruan, J. Nanomater., 2014, 2014, 206370.

[15] W. Rosenhain, J. Inst. Met., 1913, 10, 119.

[16] W. T. Read and W. Shockley, Phys. Rev., 1950, 78, 275.

[17] F. C. Frank, Report of the Symposium on the Plastic Deformation of Crystalline Solids, Carnegie Institute of Technology, Pittsburgh, 1950, 150.

[18] P. Klemens, Proc. Phys. Soc. A, 1955, 68, 1113.

[19] D. Xu, R. Hanus, Y. Xiao, S. Wang, G. J. Snyder and Q. Hao, Mater. Today Phys., 2018, 6, 53-59.

[20] R. Hanus, A. Garg and G. J. Snyder, Commun. Phys., 2018, 1, 1-11.

[21] M. G. Tsoutsouva, P. E. Vullum, K. Adamczyk, M. D. Sabatino and G. Stokkan, J. Appl. Phys. 2020, 127, 125109.

[22] J. Mao, Y. Wang, Z. Liu, B. Ge and Z. Ren, Nano Energy, 2017, 32, 174-179.

[23] G. Chen, Nanoscale En. Transport and Conversion: A Parallel Treatment of Electrons, Molecules, Phonons, and Photons., Oxford University Press, New York, 2005.
[24] R. M. Costescu, M. A. Wall and D. G. Cahill, Phys. Rev. B, 2003, 67, 054302.

[25] C. Monachon, L. Weber and C. Dames, Annu. Rev. Mater. Res., 2016, 46, 433-463.

[26] I. M. Khalatnikov, Zhurnal Eksperimentalnoi Teor. Fiz., 1952, 22, 687-704.

[27] R. M. Mazo, Theoretical studies on low temperature phenomena, PhD thesis, Yale University, 1955.

[28] W. Little, Can. J. Phys., 1959, 37, 334-349.

[29] C. B. Saltonstall, C. A. Polanco, J. C. Duda, A. W. Ghosh, P. M. Norris and P. E. Hopkins, J. Appl. Phys., 2013, 113, 013516.

[30]R. Prasher, Appl. Phys. Lett., 2009, 94, 041905.

[31] E. T. Swartz and R. O. Pohl, Appl. Phys. Lett., 1987, 51, 2200-2202.

[32] P. Reddy, K. Castelino and A. Majumdar, Appl. Phys. Lett., 2005, 87, 211908.

[33] D. Singh, J. Y. Murthy and T. S. Fisher, J. Heat Transfer, 2010, 133, 122401 .

[34] Q. Hao, G. Zhu, G. Joshi, X. Wang, A. Minnich, Z. Ren and G. Chen, Appl. Phys. Lett., 2010, 97, 063109.

[35] P. K. Schelling, S. R. Phillpot and P. Keblinski, J. Appl. Phys., 2004, 95, 6082-6091.

[36] S. H. Ju and X. G. Liang, J. Appl. Phys., 2013, 113, 053513053517.

[37] Z. Zheng, X. Chen, B. Deng, A. Chernatynskiy, S. Yang, L. Xiong and Y. Chen, J. Appl. Phys., 2014, 116, 073706.

[38] Z. Tian, K. Esfarjani and G. Chen, Phys. Rev. B, 2012, 86, 235304.

[39] Z. Tian, K. Esfarjani and G. Chen, Bull. Am. Phys. Soc., 2012, 57.

[40] W. Zhang, T. Fisher and N. Mingo, Numerical Heat Transfer, Part B: Fundamentals, 2007, 51, 333-349.

[41] X. Li and R. Yang, Phys. Rev. B, 2012, 86, 054305.

[42] W. Zhang, T. Fisher and N. Mingo, J. Heat transfer, 2007, 129, 483-491.

[43] H. Zhao and J. B. Freund, J. Appl. Phys., 2009, 105, 013515.

[44] Z. Wang, J. E. Alaniz, W. Jang, J. E. Garay and C. Dames, Nano Lett., 2011, 11, 2206-2213.

[45] P. E. Hopkins, ISRN Mech. Eng., 2013, 682586.

[46] R. S. Prasher, P. E. Phelan, J Heat Transfer, 2001,123, 105112.

[47] T. Beechem, S. Graham, P. Hopkins, P. Norris, Appl. Phys. Lett., 2007, 90, 054104.

[48] Y . Zhang, D. Ma, Y. Zang, X. Wang and N. Yang, Front. Energy Res., 2018, 6.

[49] X. Meng, W. Cai, Z. Liu, J. Li, H. Geng and J. Sui, Acta Mater, 2015, 98, 405-415.

[50] A. Bagri, S.-P. Kim, R. S. Ruoff and V. B. Shenoy, Nano lett., 2011, 11, 3917-3921.

[51] H. Dong, J. Xiao, R. Melnik and B. Wen, Sci. Rep., 2016, 6 (1), 19575.

[52] P. Klemens, Int. J. Thermophys., 1994, 15, 1345-1351.

[53] H. S. Kim, S. I. Kim, K. H. Lee, S. W. Kim and G. J. Snyder, Phys. Status Solidi B, 2017, 254, 1600103. 
[54] H.-S. Kim, S. D. Kang, Y. Tang, R. Hanus and G. Jeffrey Snyder, Mater. Horiz., 2016, 3, 234-240.

[55] J. M. Ziman, Electrons and phonons: the theory of transport phenomena in solids., Oxford University Press, 2001.

[56] M. Omini and A. Sparavigna, Phys. Rev. B, 2000, 61, 6677. [57] P. Carruthers, Phys. Rev., 1959, 114, 995.

[58] M. Li, Z. Ding, Q. Meng, J. Zhou, Y. Zhu, H. Liu, M. S. Dresselhaus and G. Chen, Nano Lett., 2017, 17, 1587-1594.

[59] R. Gurunathan, R. Hanus, S. Graham, A. Garg and G. J. Snyder, Phys. Rev. B, 2021, 103, 144302.

[60] Z. Liang and M. Hu, J. Appl. Phys., 2018, 123, 191101. [61]A. Maiti, G. Mahan and S. Pantelides, Solid State Commun., 1997, 102, 517-521.

[62] C. Li and Z. Tian, Front. Phys., 2019, 7, 3.

[63] Y. Lu and J. Guo, App. Phys. Lett., 2012, 101, 043112.

[64] M. Khalkhali, A. Rajabpour and F. Khoeini, Sci. rep., 2019, 9, 1-12.

[65] L. M. Sandonas, H. Sevinçli, R. Gutierrez and G. Cuniberti, Adv. Sci., 2018, 5, 1700365.

[66] Y. Sun, Y. Zhou, J. Han, W. Liu, C. Nan, Y. Lin, M. Hu and B. Xu, Npj Comput. Mater, 2019, 5, 97.

[67] Y. Sun, Y. Zhou, J. Han, M. Hu, B. Xu, and W. Liuet, $J$. Appl. Phys., 2020, 127, 045106.

[68] Y. Sun, Y. Zhou, M. Hu G. J. Snyder, B. Xu, and W. Liu, J. Appl. Phys., 2021, 129, 055103.

[69] N. Yang, T. Luo, K. Esfarjani, A. Henry, Z. Tian, J. Shiomi, Y. Chalopin, B. Li and G. Chen, J. Comput. Theor. Nanosci. 2015, 12, 168-174.

[70] E. Lee and T. Luo, Phys. Chem. Chem. Phys., 2017, 19, 18407-18415.

[71] K. Gordiz and A. Henry, Scientific Reports, 2016, 6, 23139.

[72] X. Tian and M. Chen, Sci. rep., 2021, 11, 739-739.

[73] Y.-J. Wu, L. Fang and Y. Xu, Npj Comput. Mater., 2019, 5, $1-8$.

[74] S. Fujii, T. Yokoi, C. A. J. Fisher, H. Moriwake and M. Yoshiya, Nat. Commun., 2020, 11, 1854.

[75] H. Zhang, K. Hippalgaonkar, T. Buonassisi, O. M. L. LÃ,vvik, E. Sagvolden and D. Ding, ES Energy \& Eniron., 2018, 2, 1-8.

[76] S. Fujii, T. Yokoi and M. Yoshiya, Acta Mater., 2019, 171, 154-162.

[77] C. W. Rosenbrock, E. R. Homer, G. Csányi and G. L. W. Hart, npj Computational Mat.s, 2017, 3, 29.

[78] J. L. Priedeman, C. W. Rosenbrock, O. K. Johnson and E. R. Homer, Acta Mater., 2018, 161, 431-443.

[79] A. J. Minnich, M. S. Dresselhaus, Z. F. Ren and G. Chen, Energy Environ. Sci., 2009, 2, 466-479.

[80] B. Poudel, Q. Hao, Y. Ma, Y. Lan, A. Minnich, B. Yu, X. Yan, D. Wang, A. Muto, D. Vashaee, X. Chen, J. Liu, M. S. Dresselhaus, G. Chen and Z. Ren, Science, 2008, 320, 634-638.

[81] Z. Liu, J. Mao, T.-H. Liu, G. Chen and Z. Ren, MRS Bulletin, 2018, 43, 181-186.

[82] H. Casimir, Physica, 1938, 5, 495-500.

[83] Q. Hao, J. Appl. Phys., 2012, 111, 014307.

[84] T. Zeng and G. Chen, J. Heat Transfer, 2001, 123, 340-347.
[85] C. Dames and G. Chen, J. Appl. Phys., 2004, 95, 682-693.

[86] L. Braginsky, V. Shklover, H. Hofmann and P. Bowen, Phys. Rev. B, 2004, 70, 134201.

[87] Q. Hao, J. Appl. Phys., 2014, 116, 034305.

[88] Q. Hao, H. Zhao, Y. Xiao and D. Xu, J. Appl. Phys., 2018, 123, 014303 .

[89] P. L. Palla and S. Giordano, J. Appl. Phys., 2016, 120, 184301.

[90] M. Maldovan, J. Appl. Phys., 2011, 110, 114310.

[91] F. Badry and K. Ahmed, AIP Advances, 2020, 10, 105021.

[92] D. S. Smith, S. Fayette, S. Grandjean, C. Martin, R. Telle and T. Tonnessen, J. Am. Ceram. Soc., 2003, 86, 105-111.

[93] C.-W. Nan and R. Birringer, Phy. Rev. B, 1998, 57, 8264.

[94] H. Dong, B. Wen and R. Melnik, Sci. Rep., 2014, 4, 7037.

[95] T. Hori, J. Shiomi and C. Dames, Appl. Phy. Lett., 2015, 106, 171901 .

[96] Q. Hao, Y. Xiao and H. Zhao, Appl. Therm. Eng., 2017, 111, 1409-1416.

[97] M. Takashiri, S. Tanaka, H. Hagino and K. Miyazaki, J. Appl. Phy., 2012, 112, 084315.

[98] K. Watari, H. Nakano, K. Urabe, K. Ishizaki, S. Cao and K. Mori, J. Mater. Res., 2002, 17, 2940-2944.

[99] R. Berman, Proc. Phys. Soc. A., 1952, 65, 1029.

[100] M. Takashiri, K. Miyazaki, S. Tanaka, J. Kurosaki, D. Nagai and H. Tsukamoto, J. Appl. Phys., 2008, 104, 084302.

[101] C.-W. Nan, Prog. Mater. Sci., 1993, 37, 1-116.

[102] Y. Lan, B. Poudel, Y. Ma, D. Wang, M. S. Dresselhaus, G. Chen and Z. Ren, Nano Lett., 2009, 9, 1419-1422.

[103] Q. Hao, J. Appl. Phys., 2012, 111, 014309.

[104] M.-S. Jeng, R. Yang, D. Song and G. Chen, J. Heat Transfer, 2008, 130.

[105] Y. Xiao, Q. Chen, D. Ma, N. Yang and Q. Hao, arXiv preprint arXiv:1910.04913, 2019.

[106] A. Minnich and G. Chen, App. Phys. Lett., 2007, 91, 073105.

[107] I. T. Witting, T. C. Chasapis, F. Ricci, M. Peters, N. A. Heinz, G. Hautier and G. J. Snyder, Adv. Electron. Mater., 2019, 5, 1800904.

[108] H. Goldsmid, J. Appl. Phy., 1961, 32, 2198-2202.

[109] E. A. Mityushov, R. A. Adamesku and P. V. Gel'd, J. Eng. Phys., 1984, 47, 1052-1056.

[110] F. Yang, T. Ikeda, G. J. Snyder and C. Dames, J. Appl. Phy., 2010, 108, 034310.

[111] J. Ma and C. Nan, Ann. Rev. Heat Transfer, 2014, 17.

[112] J. Shen, L. Hu, T. Zhu and X. Zhao, App. Phy. Lett., 2011, 99 (12), 124102.

[113] J. J. Shen, T. J. Zhu, X. B. Zhao, S. N. Zhang, S. H. Yang and Z. Z. Yin, Energy Environ. Sci., 2010, 3, 1519-1523.

[114] O. Ben-Yehuda, R. Shuker, Y. Gelbstein, Z. Dashevsky and M. P. Dariel, J. Appl. Phys., 2007, 101, 113707.

[115] X. Yan, B. Poudel, Y. Ma, W. Liu, G. Joshi, H. Wang, Y. Lan, D. Wang, G. Chen and Z. Ren, Nano Lett., 2010, 10, 33733378.

[116] Y. Pan and J. F. Li, NPG Asia Mater., 2016, 8, e275-e275. 
[117] K. Biswas, J. He, I. D. Blum, C.-I. Wu, T. P. Hogan, D. N. Seidman, V. P. Dravid and M. G. Kanatzidis, Nature, 2012, 489, 414-418.

[118] S. I. Kim, K. H. Lee, H. A. Mun, H. S. Kim, S. W. Hwang, J. W. Roh, D. J. Yang, W. H. Shin, X. S. Li and Y. H. Lee, Science, 2015, 348, 109-114.

[119] L.-D. Zhao, V. P. Dravid and M. G. Kanatzidis, Energy Environ. Sci., 2014, 7, 251-268.

[120] T. Hori and J. Shiomi, Sci. Technol. Adv. Mater., 2019, 20, $10-25$.

[121] H. S. Kim, S. I. Kim, K. H. Lee, S. W. Kim and G. J. Snyder, Phys. Status Solidi B, 2017, 254, 1600103.

[122] A. Kundu, N. Mingo, D. A. Broido and D. A. Stewart, Phys. Rev. $B, 2011,84,125426$.

[123] A. Majumdar, J. Heat Transf., 1993, 115.

[124] N. Mingo, D. Hauser, N. P. Kobayashi, M. Plissonnier and A. Shakouri, Nano Lett., 2009, 9, 711-715.

[125] H. Zhang and A. J. Minnich, Sci. Rep., 2015, 5, 8995-8995. [126] C.-W. Nan, R. Birringer, D. R. Clarke and H. Gleiter, J. Appl. Phys., 1997, 81, 6692-6699.

[127] Q. Hao, Y. Xiao and H. Zhao, Appl. Therm. Eng., 2016, 111, 1409.

[128] D. Xu, Q. Wang, X. Wu, J. Zhu, H. Zhao, B. Xiao, X. Wang, X. Wang and Q. Hao, Front. Energy, 2018, 12, 127-136.

[129] S. Alaie, D. F. Goettler, M. Su, Z. C. Leseman, C. M. Reinke and I. El-Kady, Nat. Commun., 2015, 6, 7228.

[130] C. Huang, X. Zhao, K. Regner and R. Yang, Physica E Low Dimens. Syst. Nanostruct., 2018, 97, 277-281.

[131] P. E. Hopkins, P. T. Rakich, R. H. Olsson, I. F. El-Kady and L. M. Phinney, Appl. Phys. Lett., 2009, 95, 161902.

[132] P. E. Hopkins, L. M. Phinney, P. T. Rakich, R. H. Olsson and I. El-Kady, Appl. Phys. A, 2011, 103, 575-579.

[133] A. Sarua, H. Ji, K. Hilton, D. Wallis, M. J. Uren, T. Martin and M. Kuball, IEEE Trans. Electron Dev., 2007, 54, 3152-3158. [134] P. E. Hopkins, J. C. Duda, S. P. Clark, C. P. Hains, T. J. Rotter, L. M. Phinney and G. Balakrishnan, Appl. Phys. Lett., 2011, 98, 161913.

[135] S. Deng, C. Xiao, J. Yuan, D. Ma, J. Li, N. Yang and H. He, Appl. Phys. Lett., 2019, 115, 101603.

[136] E. K. Kim, S. I. Kwun, S. M. Lee, H. Seo and J. G. Yoon, Appl. Phys. Lett., 2000, 76, 3864-3866.

[137] J. Yang, Q. Hao, H. Wang, Y. C. Lan, Q. Y. He, A. Minnich, D. Z. Wang, J. A. Harriman, V. M. Varki, M. S. Dresselhaus, G. Chen and Z. F. Ren, Phys. Rev. B, 2009, 80, 115329.

[138] K. Tai, A. Lawrence, M. P. Harmer and S. J. Dillon, Appl. Phy. Lett., 2013, 102, 034101.

[139] D. H. Hurley, M. Khafizov and S. Shinde, J. of App. Phy., 2011, 109, 083504.

[140] J. Han, S. L. Thomas and D. J. Srolovitz, Prog. in Mat. Sci., 2018, 98, 386-476.

[141] L. Sun, M. A. L. Marques and S. Botti, Nat. Comm., 2021, 12,811 .

[142] D. Wolf, in Handbook of Material Modeling, Springer, 2005, pp. 1953-1983.
[143] H. Liu, Y. Lin and S. Luo, J. Phys. Chem. C, 2014, 118 , 24797-24802

[144] T. Watanabe, B. Ni, S. R. Phillpot, P. K. Schelling and P. Keblinski, J. Appl. Phys., 2007, 102, 063503.

[145] Q. Meng, L. Wu and Y. Zhu, Phys. Rev. B, 2013, 87, 064102. [146]Y. Xue, Y. Chen, K. Cai, Z.-Y. Liu, Y. Zhang and N. Wei, Appl. Phys. Lett., 112, 021604.

[147] J. Song, Z. Xu, X. He, C. Cai, Y. Bai, L. Miao and R. Wang, Phys. Chem. Chem. Phy., 2020, 22, 11537-11545.

[148] S. Wang, D. Xu, R. Gurunathan, G. J. Snyder and Q. Hao, J. Materiomics, 2020, 6, 248-255.

[149] A. Otsuki, Interface Sci., 2001, 9, 293-296.

[150] N. Kawamoto, Y. Kakefuda, T. Mori, K. Hirose, M. Mitome, Y. Bando and D. Golberg, Nanotechnology, 2015, 26, 465705.

[151] P. Yasaei, A. Fathizadeh, R. Hantehzadeh, A. K. Majee, A. El-Ghandour, D. Estrada, C. Foster, Z. Aksamija, F. KhaliliAraghi and A. Salehi-Khojin, Nano lett., 2015, 15, 4532-4540.

[152] A. Sood, R. Cheaito, T. Bai, H. Kwon, Y. Wang, C. Li, L. Yates, T. Bougher, S. Graham, M. Asheghi, M. Goorsky and K. E. Goodson, Nano Lett., 2018, 18, 3466-3472.

[153] H. Mun, S. M. Choi, K. H. Lee and S. W. Kim, ChemSusChem, 2015, 8, 2312-2326.

[154] L. Yang, X. Wan, D. Ma, Y. Jiang and N. Yang, Phys. Rev. $B, 2021, \mathbf{1 0 3}, 155305$.

[155] Z. Liang, K. Sasikumar and P. Keblinski, Phys. Rev. Lett., 2014, 113, 065901.

[156] J. C. Duda and P. E. Hopkins, App. Phy. Lett., 2012, 100, 111602.

[157] X. W. Zhou, R. E. Jones, C. J. Kimmer, J. C. Duda and P. E. Hopkins, Physical Rev. B, 2013, 87, 094303.

[158] P. E. Hopkins, J. C. Duda, C. W. Petz and J. A. Floro, Phys. Rev. $B, 2011, \mathbf{8 4}, 035438$.

[159] E. Lee, T. Zhang, T. Yoo, Z. Guo and T. Luo, ACS Appl. Mater. Interfaces, 2016, 8, 35505-35512.

[160] W. Park, A. Sood, J. Park, M. Asheghi, R. Sinclair and K. E. Goodson, Nanoscale Microscale Thermophys. Eng., 2017, 21, 134-144.

[161] E. Lee and T. Luo, Appl. Phys. Lett., 2018, 112, 011603.

[162] J. Cho, Z. Li, E. Bozorg-Grayeli, T. Kodama, D. Francis, F. Ejeckam, F. Faili, M. Asheghi and K. E. Goodson, IEEE Trans. Compon. Packaging Manuf. Technol., 2013, 3, 79-85.

[163] T. S. English, J. C. Duda, J. L. Smoyer, D. A. Jordan, P. M. Norris and L. V. Zhigilei, Phys. Rev. B, 2012, 85, 035438.

[164] H. J. Goldsmid, Thermoelectric Refrigeration, Plenum, New York, 1964

[165] X. Ji, J. He, Z. Su, N. Gothard and T. M. Tritt, J. Appl. Phys., 2008, 104, 034907.

[166] S. K. Bux, R. G. Blair, P. K. Gogna, H. Lee, G. Chen, M. S. Dresselhaus, R. B. Kaner and J. P. Fleurial, Adv. Funct. Mater., 2009, 19, 2445-2452.

[167] S. Jo, S. H. Park, H. W. Ban, D. H. Gu, B.-S. Kim, J. H. Son, H.-K. Hong, Z. Lee, H.-S. Han and W. Jo, J. Alloys Compd., 2016, 689, 899-907. 
[168] R. Deng, X. Su, Z. Zheng, W. Liu, Y. Yan, Q. Zhang, V. P. Dravid, C. Uher, M. G. Kanatzidis and X. Tang, Sci. Adv., 2018, 4, eaar5606.

[169] Z. Chen, Z. Jian, W. Li, Y. Chang, B. Ge, R. Hanus, J. Yang, Y. Chen, M. Huang, G. J. Snyder and Y. Pei, Adv. Mater., 2017, 29, 1606768.

[170] P. A. Zong, R. Hanus, M. Dylla, Y. Tang, J. Liao, Q. Zhang, G. J. Snyder and L. Chen, Energy Environ. Sci., 2017, 10, 183 191.

[171] H. Li, X. Su, X. Tang, Q. Zhang, C. Uher, G. J. Snyder and U. Aydemir, J. Materiomics, 2017, 3, 273-279.

[172] Q. Zhang, Z. Zhou, M. Dylla, M. T. Agne, Y. Pei, L. Wang, Y. Tang, J. Liao, J. Li, S. Bai, W. Jiang, L. Chen and G. Jeffrey Snyder, Nano Energy, 2017, 41, 501-510.

[173] A. Stoffers, B. Ziebarth, J. Barthel, O. Cojocaru-Mirédin, C. Elsässer and D. Raabe, Phys. Rev. lett., 2015, 115, 235502.

[174] S. Paciornik, R. Kilaas, J. Turner and U. Dahmen, Ultramicroscopy, 1996, 62, 15-27.

[175] Y. Won, J. Cho, D. Agonafer, M. Asheghi and K. E. Goodson, IEEE Trans. Compon. Packaging Manuf. Technol., 2015, 5, 737-744.

[176] K. W. Jung, C. R. Kharangate, H. Lee, J. Palko, F. Zhou, M. Asheghi, E. M. Dede and K. E. Goodson, Int. J. Heat Mass Transf., 2019, 130, 1108-1119.

[177] R. van Erp, R. Soleimanzadeh, L. Nela, G. Kampitsis and E. Matioli, Nature, 2020, 585, 211-216.

[178] F. Zhou, S. N. Joshi, Y. Liu and E. M. Dede, Int. Commun. Heat Mass, 2019, 108, 104300.

[179] Z. Cheng, F. Mu, L. Yates, T. Suga and S. Graham, ACS Appl. Mater. Interfaces, 2020 12, 8376-8384.

[180] L. Tao, S. Theruvakkattil Sreenivasan and R. Shahsavari, ACS Appl. Mater. Interfaces, 2017, 9, 989-998.

[181] E. Lee, T. Zhang, M. Hu and T. Luo, Phys. Chem. Chem. Phys., 2016, 18, 16794-16801.

[182] M. Hu, X. Zhang, D. Poulikakos and C. P. Grigoropoulos, Int. J. Heat Mass Transf., 2011, 54, 5183-5191.

[183] X. Zhao, X. Qian, X. Li, R. Yang, J. Appl. Phys., 2021, 129, 215105.

[184] F. Wakai, N. Enomoto and H. Ogawa, Acta Mater, 2000, 48, 1297-1311.

\section{Author information}

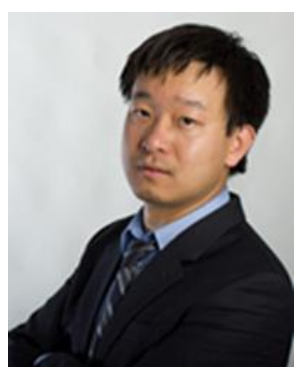

Qing Hao is an Associate Professor of Department of Aerospace and Mechanical Engineering at the University of Arizona. His research focuses on nanoscale energy transport in materials and electronic devices, including thermoelectrics, high-power electronics, and 2D materials. He received the $2008 R \& D 100$ Award as a team member for thermoelectrics research, 2015 AFOSR YIP Award for graphene studies, and 2017 NSF CAREER Award for thermal studies of grain boundaries. He was selected as the inaugural class of the Craig M. Berge Dean's Fellows in 2020.

Publisher's Note Engineered Science Publisher remains neutral with regard to jurisdictional claims in published maps and institutional affiliations. 